\title{
The Effects of Spaceflight Microgravity on the Musculoskeletal System of Humans and Animals, with an Emphasis on Exercise as a Countermeasure: A Systematic Scoping Review
}

\author{
Darya MOOSAVI ${ }^{1}$, David WOLOVSKY ${ }^{1}$, Angela DEPOMPEIS ${ }^{1}$, David UHER ${ }^{1}$, David \\ LENNINGTON ${ }^{1}$, Roddy BODDEN ${ }^{1}$, Carol Ewing GARBER ${ }^{1}$ \\ ${ }^{1}$ Department of Biobehavioral Sciences, Teachers College, Columbia University. New York City, \\ NY, United States
}

Received July 29, 2020

Accepted February 18, 2021

\section{Summary}

The purpose of this systematic review is twofold: 1) to identify, evaluate, and synthesize the heretofore disparate scientific literatures regarding the effects of direct exposure to microgravity on the musculoskeletal system, taking into account for the first time both bone and muscle systems of both humans and animals; and 2) to investigate the efficacy and limitations of exercise countermeasures on the musculoskeletal system under microgravity in humans. The Framework for Scoping Studies (Arksey and O'Malley 2005) and the Cochrane Handbook for Systematic Reviews of Interventions (Higgins JPT 2011) were used to guide this review. The Preferred Reporting Items for Systematic Reviews and Meta-Analyses (PRISMA) checklist was utilized in obtaining the combined results (Moher, Liberati et al. 2009). Data sources, PubMed, Embase, Scopus, and Web of Science were searched for published articles through October 2019 using the Mesh terms of microgravity, musculoskeletal system, and exercise countermeasures. A total of 84 references were selected, including 40 animal studies and 44 studies with human participants. The heterogeneity in the study designs, methodologies, and outcomes deemed this review unsuitable for a meta-analysis. Thus, we present a narrative synthesis of the results for the key domains under five categories:

1) Skeletal muscle responses to microgravity in humans

2) Skeletal muscle responses to microgravity in animals

3) Adaptation of the skeletal system to microgravity in humans

4) Adaptation of the skeletal system to microgravity in animals

5) Effectiveness of exercise countermeasures on the human musculoskeletal system in microgravity.
Existing studies have produced only limited data on the combined effects on bone and muscle of human spaceflight, despite the likelihood that the effects on these two systems are complicated due to the components of the musculoskeletal system being anatomically and functionally interconnected. Bone is directly affected by muscle atrophy as well as by changes in muscle strength, notably at muscle attachments. Given this interplay, the most effective exercise countermeasure is likely to be robust, individualized, resistive exercise, primarily targeting muscle mass and strength.

\section{Key words}

Microgravity • Musculoskeletal system • Exercise countermeasure

- Spaceflight • Skeleton • Skeletal Muscle

\section{Corresponding author}

Darya Moosavi, 525 W 120th St, New York, NY 10027, USA. E-mail: zam2113@tc.columbia.edu

\section{Introduction}

With the prospect of sending humans to Mars (and beyond), understanding the effects of physiological changes on the health of astronauts is critical for successful long-duration spaceflight (SF). Prolonged exposure to microgravity $(\mu \mathrm{G})$, is harmful to the bones and muscles of the human body. When astronauts are in a $\mu \mathrm{G}$ environment, they experience decreases in bone density and muscle volume, as well as changes in muscle 
fiber properties (Trappe et al. 2009). A weakened musculoskeletal system comprises the ability of astronauts to perform mission-critical tasks both during spaceflight and upon reentry to a gravitational environment. While various exercise countermeasures have been evaluated, one single successful method in maintaining the integrity of the musculoskeletal system on long-duration spaceflight has not yet been found. Since skeletal muscles, tendons, ligaments, and bones are anatomically and functionally interconnected, the reductionist approach that focuses on only one part of this complex system is inadequate for determining the most protective exercise protocols for astronauts. This systematic review thus aims to synthesize for the first time the scientific literature regarding the effects of $\mu \mathrm{G}$ on both bone and muscle, describe exercise protocols that have been used in $\mu \mathrm{G}$, and identify crucial features of effective countermeasures to SF-induced atrophy. We review studies based on direct exposure to $\mu \mathrm{G}$ in both humans and animals. This topic is of particular relevance, especially amid the coronavirus (COVID-19) pandemic. Patients with COVID-19 sustain muscle atrophy due to hospital-associated deconditioning. Likewise, astronauts experience muscular deterioration from long-term inactivity and muscle unloading in the microgravity environment.

\section{Purpose}

The purpose of this systematic review is twofold: 1) to identify, evaluate, and synthesize heretofore disparate scientific literatures regarding the effects of direct exposure to microgravity on the musculoskeletal system, taking into account for the first time both bone and muscle systems of both humans and animals; and 2) to investigate the efficacy and limitations of exercise countermeasures on the musculoskeletal system under microgravity in humans.

\section{Methods}

This systematic review was conducted in accordance with the methodological framework for scoping studies (Arksey and O'Malley 2005) and the guidelines of the Cochrane Handbook for Systematic Reviews of Interventions (Higgins JPT 2011) to ensure a transparent and complete investigation. Also, the PRISMA (Preferred Reporting Items for Systematic Reviews and Meta-analyses) checklist was used to report the results (Moher et al. 2009). The scoping methodology was employed because our research questions were broad and complex. Additionally, this topic covers several different study designs that could not be identified in advance.

Table 1. PICOS eligibility criteria model

\begin{tabular}{|c|c|c|}
\hline Parameter & Inclusion criteria & Exclusion criteria \\
\hline Population & $\begin{array}{l}\text { Astronauts/cosmonauts and animals with no sex or age } \\
\text { restrictions }\end{array}$ & $\begin{array}{l}\text { Populations with no direct exposure to } \\
\text { spaceflight conditions }\end{array}$ \\
\hline $\begin{array}{l}\text { Intervention or } \\
\text { Exposure }\end{array}$ & $\begin{array}{l}\text { Direct exposure to } \mu \mathrm{G} \text { for at least } 24 \text { hours or longer } \\
\text { Countermeasure: physical activity/exercise training, } \\
\text { including resistance training }\end{array}$ & $\begin{array}{l}\text { All other analogs such as bed rest, hind limb } \\
\text { suspension, immobilization, disuse, and } \\
\text { parabolic flights } \\
\text { All other countermeasures including } \\
\text { pharmacologic or dietary supplementation, } \\
\text { electrical stimulation, hybrid, artificial gravity }\end{array}$ \\
\hline Comparator & $\begin{array}{l}\text { No exposure to } \mu \mathrm{G} \text { (e.g., ground-based controls, bed rest, } \\
\text { etc.) } \\
\text { No exercise countermeasure }\end{array}$ & N/A \\
\hline Outcomes & $\begin{array}{l}\text { Physiological changes in skeletal muscle (e.g., muscle } \\
\text { mass, strength, fiber types, etc.), and/or skeletal system } \\
\text { (e.g., bone density, structure, such as cortical thickness or } \\
\text { porosity) } \\
\text { Physiological effects of exercise countermeasures }\end{array}$ & $\begin{array}{l}\text { No defined physiological outcomes related to the } \\
\text { musculoskeletal systém } \\
\text { All other non-physiological outcomes } \\
\text { (e.g., biomechanical and motor control) }\end{array}$ \\
\hline Study Design & $\begin{array}{l}\text { Primary studies including } \\
\text { RCT, NRCT, and single group studies }\end{array}$ & $\begin{array}{l}\text { All nonexperimental designs including } \\
\text { editorials, opinions, pilot studies, narrative } \\
\text { reviews, meta-analyses }\end{array}$ \\
\hline
\end{tabular}


Table 2. Search strategy

\begin{tabular}{lll}
\hline Search \# & Keywords & Terms and MeSH vocabulary \\
\hline 1 & Microgravity & Microgravity OR spaceflight OR weightlessness \\
\hline 2 & Musculoskeletal & Muscle OR bone OR musculoskeletal OR 'skeletal muscle' \\
\hline 3 & Countermeasure & Exercise OR training OR countermeasure \\
\hline 4 & Combined search & 1 AND 2 AND 3 \\
& 1 AND 2 \\
& 1 AND 3 \\
\hline
\end{tabular}

Note. Medical Subject Headings (MeSH) as a comprehensive controlled vocabulary for the purpose of indexing journal articles and books in the life sciences were included in the search strategy. In the Pubmed advanced search builder either 'Title/Abstract' [tiab] was used.

\section{Eligibility criteria}

Inclusion criteria included the population, intervention, comparison, outcome, and study design (PICOS) model (Richardson 1995). The PICOS eligibility criteria are presented in Table 1.

\section{Search strategy for identification of studies}

The electronic searches were performed in August 2018, by the first author with the assistance of a research librarian from the Augustus C. Long Health Sciences Library of Columbia University.

The literature search was conducted in English. A range of medical subject headings (MeSH) (or equivalent structured terms in other databases) and keywords related to $\mu \mathrm{G}$, the musculoskeletal system, and exercise were used in various combinations. Searches were performed on PubMed, Embase, Scopus, and Web of Science (Table 2). The search strategy was specifically tailored to each database without applying date restrictions. The searches in the databases were repeated in October 2019. Relevant studies from this search were then added into the formal review procedure. To ensure literature saturation, forward and backward citation tracking were employed for key review articles and eligible articles identified through the electronic search. A third search was performed in December 2020, which included the term "hypogravity" to ensure that no relevant studies using this term had been omitted. In this review we use the term "microgravity" rather than "hypogravity" because the former term was used more often in the studies that met our inclusion criteria, but this should not be interpreted as a methodological rejection of the term "hypogravity" or studies using it.

\section{Risk of bias in individual studies}

The Cochrane Collaboration's tool for assessing risk of bias was used to assess the quality of included studies (Moher et al. 2009). Two authors independently assessed the risk of bias. Uncertainties or disagreements were resolved by consensus or discussed with a third reviewer when necessary. Risk of bias was evaluated as low, high, or unclear, according to the following factors: random sequence generation, allocation concealment, blinding of participants and personnel, blinding of outcome assessment, incomplete outcome data, and selective outcome data, as well as other sources of bias.

\section{Results}

\section{Study selection}

The search of PubMed, Embase, Scopus, and Web of Science databases provided a total of 3,255 citations. An additional 471 citations were identified by backward search (checking the references of review papers) and repeating database searches after one year. All relevant search records were initially exported to Endnote, then to Covidence Software (Covidence systematic review software, Veritas Health Innovation, Melbourne, Australia, available at www.covidence.org). After removing duplicates, 2,533 studies remained for title and abstract screening. Two authors independently screened the title and abstract of all these studies, using the Covidence software. Of these, 1,943 studies were excluded because they clearly did not meet the inclusion criteria or because the full text of the study was not 
available. The remaining 590 citations were transferred to full-text screening and reviewed by four authors for the final inclusion decision, using the PICOS criteria. At this stage, 506 studies did not meet the inclusion criteria. Ultimately, a total of 84 studies met the inclusion criteria, were extracted, and were included in the systematic review (Fig. 1). Experimental and quasi-experimental designs published in full text in peer-reviewed journals were included. Disagreements about inclusion were resolved by consensus or by the fifth author where necessary. Study characteristics are available in Tables 3 to 7 .
Data collection process

A data extraction sheet was developed by the first author using the Cochrane Consumers and Communication Review Group's data extraction template, and it was tested on four randomly selected included studies and refined accordingly. Each study was extracted by two independent reviewers. Reported items included, but were not limited to, general study information, methods of the study, risk of bias assessment, study characteristics (participants, flight duration, and countermeasures), study outcomes, data, and results.
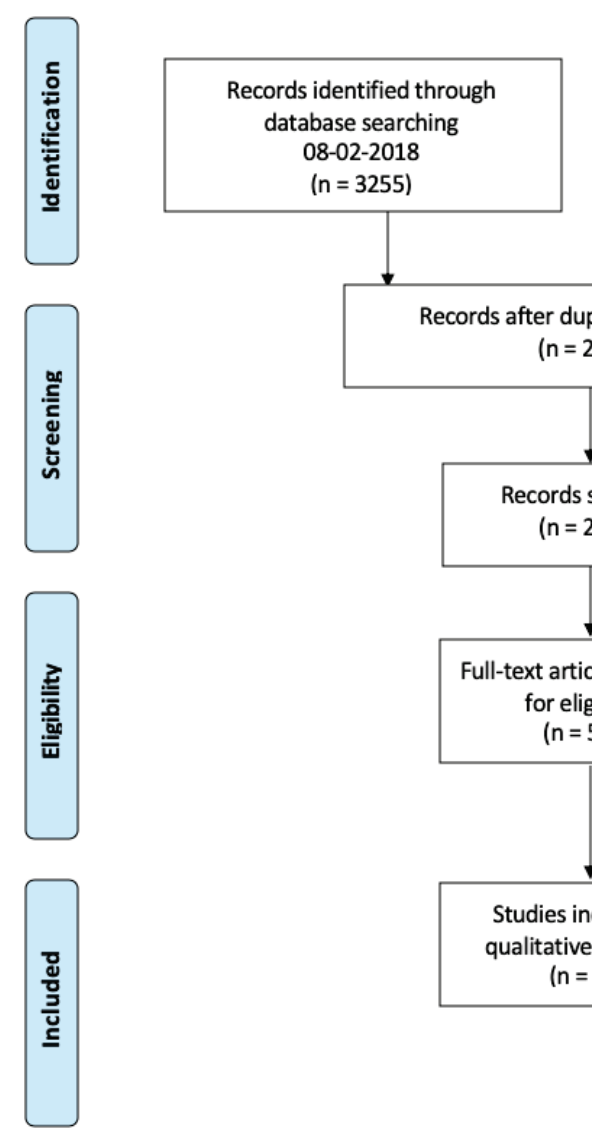

$08-02-2018$

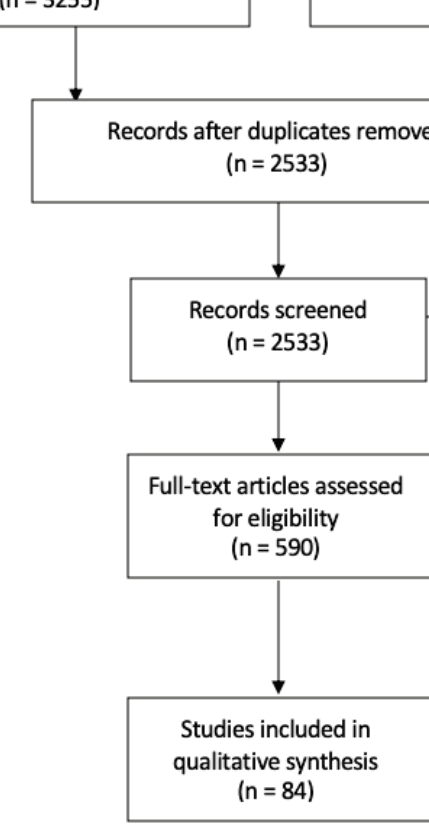

Records identified through backward search and repeating database search 10-2-2019

$$
(n=471)
$$

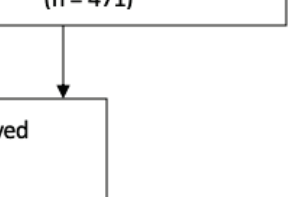

Fig. 1. PRISMA Flow Diagram

Bias arising from the randomization process

Bias due to deviations from intended interventions

Bias due to missing outcome data

Bias in measurement of the outcome

Bias in selection of the reported result

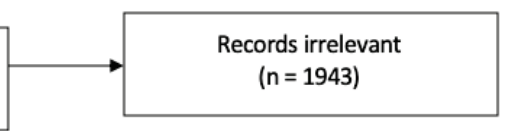

( $n=1943)$

Full-text articles excluded

( $n=506$ )

Reasons:

$(n=221)$ Full text not available ( $n=105)$ Reviews

( $n=61$ ) Wrong respond variable

(n=17) Wrong Population

( $n=15$ ) Computer modeling

( $n=13)$ Duplicate

( $n=15$ ) Device testing

( $n=14$ ) Wrong countermeasure

( $n=13)$ Wrong intervention

( $n=29)$ Wrong study

$(n=2)$ other languages

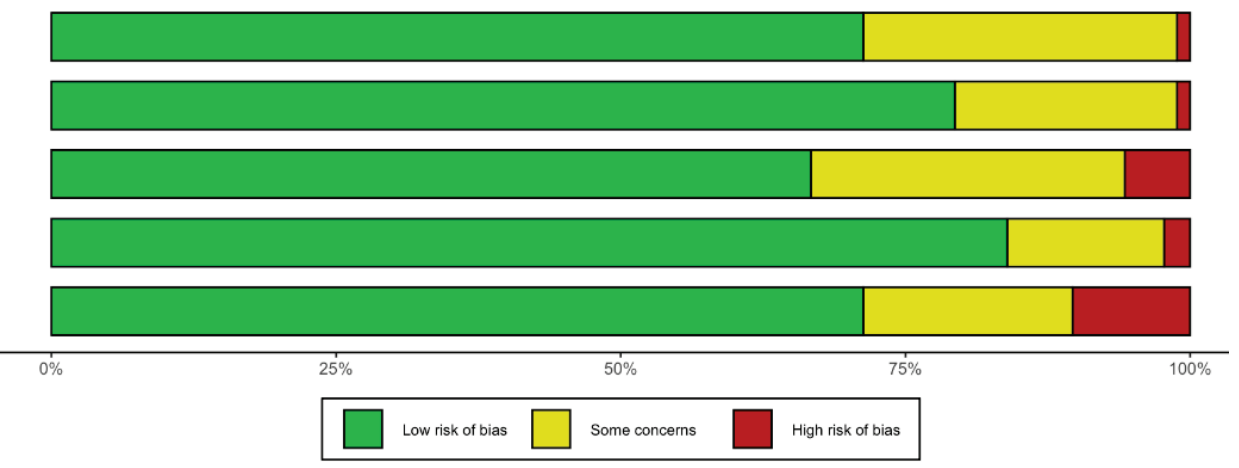

Fig. 2. Risk of bias within studies 


\section{Risk of bias in individual studies}

Quality assessment results are illustrated in Figure 2. The quality assessments were based on the articles' methods and study designs. In most studies, the methodology used was nonrandomization. In addition, the methodology of older studies, unlike more recent ones, had design flaws that might have affected the overall validity of the findings. Regarding the study designs, objective judgment of the quality of the studies was not always feasible. For example, due to the nature of space-flight investigations, single-group designs had small sample sizes and lacked control arms. Also, blinding was impractical as in most space research. Consequently, in our assessment, the lack of blinding of participants, or small sample sizes, did not necessarily cause the quality of a study to be deemed as low. Thus, the studies which were well-designed within the particular constraints of spaceflight research were rated as high quality.

\section{Synthesis of results}

The heterogeneity in study designs, methodologies, and outcomes made this review unsuitable for a statistical meta-analysis. Thus, we present a narrative synthesis of the results for the key domains under five categories: skeletal muscle responses to $\mu \mathrm{G}$ in humans (Table 3), skeletal muscle responses to $\mu \mathrm{G}$ in animals (Table 4), adaptation of the skeletal system to $\mu \mathrm{G}$ in humans (Table 5), adaptation of the skeletal system to $\mu \mathrm{G}$ in animals (Table 6), and effectiveness of exercise countermeasure on musculoskeletal system in spaceflight (Table 7). Due to the large number of studies, in the Discussion of the Result section, only the most prominent studies with highest quality are discussed.

Table 3. Skeletal muscle responses to microgravity in humans - Study Characteristics

\begin{tabular}{|c|c|c|c|c|c|c|}
\hline $\begin{array}{l}\text { Author, } \\
\text { Year }\end{array}$ & $\begin{array}{l}\text { Mission, } \\
\text { Duration }\end{array}$ & Subjects & Test \& Method & $\begin{array}{l}\text { Exercise } \\
\text { Regimen }\end{array}$ & $\begin{array}{l}\text { Anatomic } \\
\text { Site }\end{array}$ & Main Outcomes \\
\hline $\begin{array}{l}\text { Edgerton } \\
\text { et al. 1995) }\end{array}$ & $\begin{array}{l}\text { STS 32: } 5 \text { days } \\
\text { STS 33: } 5 \text { days } \\
\text { STS 34: } 11 \\
\text { days }\end{array}$ & $\begin{array}{l}\mathrm{N}=8 \\
5 \mathrm{M}, 3 \mathrm{~F}\end{array}$ & Muscle biopsy & $\begin{array}{l}\text { Aerobic } \\
\text { running: } n=3 \\
\text { No exercise: } \\
n=5\end{array}$ & $\begin{array}{l}\text { Midportion } \\
\text { of VL } \\
\text { muscle }\end{array}$ & $\begin{array}{l}\text { Short duration flights } \\
\text { affect muscle fiber } \\
\text { thickness, metabolism, \& } \\
\text { blood supply }\end{array}$ \\
\hline $\begin{array}{l}\text { (Antonutto } \\
\text { et al. 1995) }\end{array}$ & $\begin{array}{l}\text { Euromir: 94, } \\
31 \text { days }\end{array}$ & $\begin{array}{l}\mathrm{N}=1 \\
\mathrm{M}\end{array}$ & $\begin{array}{l}\text { MEP was } \\
\text { measured by a } \\
\text { MED }\end{array}$ & Unstated & Lower limbs & $\begin{array}{l}\text { Lower limbs' functional } \\
\text { capacity is negatively } \\
\text { affected by SF }\end{array}$ \\
\hline $\begin{array}{l}\text { (Antonutto } \\
\text { 1998) }\end{array}$ & $\begin{array}{l}\text { MIR 97, } \\
21 \text { days }\end{array}$ & $\begin{array}{l}\mathrm{N}=1 \\
\mathrm{M}\end{array}$ & $\begin{array}{l}\text { EMG activities, } \\
\& \text { MEP were } \\
\text { measured }\end{array}$ & Unstated & $\begin{array}{l}\text { Lower limbs } \\
\text { (RF, VL, } \\
\text { VM) }\end{array}$ & $\begin{array}{l}\text { Short duration flights } \\
\text { affect lower limbs' } \\
\text { functional capacity }\end{array}$ \\
\hline $\begin{array}{l}\text { (Antonutto } \\
\text { et al. 1999) }\end{array}$ & $\begin{array}{l}\text { Euromir } 94 \& \\
95, \\
31 \text { to } 180 \text { days }\end{array}$ & $\mathrm{N}=5$ & $\begin{array}{l}\text { Wpeak and } \\
\text { Wmean of both } \\
\text { MEP and MCP } \\
\text { were measured } \\
\text { using MED. }\end{array}$ & Unstated & Lower limbs & $\begin{array}{l}\text { SF leads to reductions in } \\
\text { muscle mass \& decreases } \\
\text { in lower limbs' functional } \\
\text { capacity }\end{array}$ \\
\hline $\begin{array}{l}\text { (Akima et } \\
\text { al. 2000) }\end{array}$ & $\begin{array}{l}\text { Unstated, } \\
9,15,16 \text { days }\end{array}$ & $\mathrm{N}=3$ & $\begin{array}{l}\text { The muscle } \\
\text { volume was } \\
\text { measured using } \\
\text { MRI }\end{array}$ & Unstated & $\begin{array}{l}\text { Knee } \\
\text { extensors, } \\
\text { knee flexors, } \\
\text { and PL }\end{array}$ & $\begin{array}{l}\text { Muscle atrophy varies } \\
\text { among individuals and } \\
\text { muscle groups }\end{array}$ \\
\hline $\begin{array}{l}\text { (Riley et al. } \\
\text { 2000) }\end{array}$ & $\begin{array}{l}\text { STS-78, } \\
17 \text { days }\end{array}$ & $\begin{array}{l}\mathrm{N}=4 \\
\mathrm{M}\end{array}$ & $\begin{array}{l}\text { Myofilament } \\
\text { density \& length } \\
\text { were determined } \\
\text { using biopsies. }\end{array}$ & $\begin{array}{l}\text { Undocumented } \\
\text { ad libitum } \\
\text { aerobic } \\
\text { exercise }\end{array}$ & SOL & $\begin{array}{l}\text { Atrophied muscle fibers } \\
\text { risk damage to sarcomeres }\end{array}$ \\
\hline $\begin{array}{l}\text { (Lambert } \\
\text { et al. 2001) }\end{array}$ & $\begin{array}{l}\text { EuroMir } 94, \\
95,98-\mathrm{E}, \& \text { EO } \\
19-24, \\
90-180 \text { days }\end{array}$ & $\mathrm{N}=14$ & $\begin{array}{l}\text { Contractile and } \\
\text { elastic } \\
\text { characteristics } \\
\text { were measured } \\
\text { using an ankle } \\
\text { ergometer and a } \\
\text { strain-gauge } \\
\text { torque transducer. }\end{array}$ & $\begin{array}{l}\text { Everyone had } \\
\text { access to a } \\
\text { bike, a } \\
\text { treadmill, and } \\
\text { a muscular } \\
\text { reinforcement } \\
\text { device. No } \\
\text { exercise data } \\
\text { was collected. }\end{array}$ & PL & $\begin{array}{l}\text { Muscle strength decreases } \\
\text { while muscle stiffness } \\
\text { increases as a result of SF }\end{array}$ \\
\hline
\end{tabular}


Table 3. (continued)

\begin{tabular}{|c|c|c|c|c|c|c|}
\hline $\begin{array}{l}\text { (Trappe et } \\
\text { al. 2001) }\end{array}$ & $\begin{array}{l}\text { STS-78, } \\
17 \text { days }\end{array}$ & $\begin{array}{l}\mathrm{N}=12 \mathrm{SF}: 4 \\
\mathrm{BR}: 8 \\
\mathrm{M}\end{array}$ & $\begin{array}{l}\text { Fiber composition } \\
\& \text { size, \& } \\
\text { contractile } \\
\text { characteristics } \\
\text { were determined } \\
\text { using biopsies \& } \\
\text { torque velocity } \\
\text { dynamometer. }\end{array}$ & $\begin{array}{l}\text { Undocumented } \\
\text { mandatory } \\
\text { exercise }\end{array}$ & GAS \& SOL & $\begin{array}{l}\text { Calf muscle function, fiber } \\
\text { size, \& enzyme activity } \\
\text { were not significantly } \\
\text { changed }\end{array}$ \\
\hline $\begin{array}{l}\text { (Tesch et al. } \\
\text { 2005) }\end{array}$ & $\begin{array}{l}\text { STS-78, } \\
17 \text { days }\end{array}$ & $\begin{array}{l}\mathrm{N}=12 \mathrm{SF}: 4 \\
\mathrm{GC}: 8 \\
\mathrm{M}\end{array}$ & $\begin{array}{l}\text { Muscle function } \\
\text { was measured by } \\
\text { MVC, concentric, } \\
\text { and eccentric } \\
\text { actions. Muscles } \\
\text { CSA were } \\
\text { measured using } \\
\text { MRI }\end{array}$ & $\begin{array}{l}\text { In-flight } \\
\text { exercise on a } \\
\text { cycle } \\
\text { ergometer. }\end{array}$ & $\begin{array}{l}\text { Knee } \\
\text { extensor/flex } \\
\text { or and gluteal } \\
\text { muscle } \\
\text { groups }\end{array}$ & $\begin{array}{l}\text { Knee extensor muscle } \\
\text { strength \& size decreased } \\
\text { due to SF }\end{array}$ \\
\hline $\begin{array}{l}\text { (Chang et al. } \\
\text { 2016) }\end{array}$ & $\begin{array}{l}\text { ISS mission, } \\
117-213 \text { days }\end{array}$ & $\begin{array}{l}\mathrm{N}=6 \\
1 \mathrm{~F}, 5 \\
\mathrm{M}\end{array}$ & $\begin{array}{l}\text { Supine lumbar } \\
\text { spine MRI }\end{array}$ & $\begin{array}{l}\text { There were } \\
\text { routine pre, in } \\
\text { and post-flight } \\
\text { exercise } \\
\text { programs }\end{array}$ & $\begin{array}{l}\text { Paraspinal } \\
\text { muscle, } \\
\text { Intervertebral } \\
\text { disc }\end{array}$ & $\begin{array}{l}\text { Lumbar paraspinal muscle } \\
\text { atrophied following long } \\
\text { duration SF }\end{array}$ \\
\hline $\begin{array}{l}\text { (Bailey et al. } \\
\text { 2018) }\end{array}$ & $\begin{array}{l}\text { ISS mission, } \\
180 \text { days }\end{array}$ & $\begin{array}{l}\mathrm{N}=6 \\
1 \mathrm{~F}, 5 \\
\mathrm{M}\end{array}$ & $\begin{array}{l}\text { Lumbar lordosis, } \\
\text { CSA, and FCSA } \\
\text { were determined } \\
\text { using MRI and } \\
\text { dynamic } \\
\text { fluoroscopy }\end{array}$ & Unstated & $\begin{array}{l}\text { Lumbar } \\
\text { extensor } \\
\text { muscles }\end{array}$ & $\begin{array}{l}\text { MF atrophy lead to lumbar } \\
\text { flattening \& increased } \\
\text { stiffness }\end{array}$ \\
\hline $\begin{array}{l}\text { (Puglia et al. } \\
\text { 2018) }\end{array}$ & $\begin{array}{l}\text { increment } 7: \\
180 \text { days } \\
\text { increment } 8: \\
191 \text { days }\end{array}$ & $\begin{array}{l}\mathrm{N}=2 \\
\mathrm{M}\end{array}$ & $\begin{array}{l}\text { Isometric } \\
\text { handgrip and } \\
\text { pinch force were } \\
\text { measured by } \\
\text { HGD and PFD } \\
\text { tools }\end{array}$ & Unstated & $\begin{array}{l}\text { Upper limbs } \\
\text { (fingers, } \\
\text { hand, wrist, } \\
\text { etc.) }\end{array}$ & $\begin{array}{l}\text { Strength \& functionality of } \\
\text { the arm \& hand decreased } \\
\text { due to long duration SF }\end{array}$ \\
\hline $\begin{array}{l}\text { (Burkhart } \\
\text { et al. 2019) }\end{array}$ & $\begin{array}{l}\text { Various ISS } \\
\text { missions, } \\
\text { Average } \\
\text { length: } 180 \\
\text { days }\end{array}$ & $\mathrm{N}=17$ & $\begin{array}{l}\text { The CSA and } \\
\text { attenuation } \\
\text { changes of } \\
\text { paraspinal } \\
\text { muscles were } \\
\text { measured using } \\
\text { CT scans }\end{array}$ & $\begin{array}{l}\text { Nine subjects } \\
\text { kept in-flight } \\
\text { workout logs. } \\
\text { Using a } \\
\text { treadmill, a } \\
\text { cycle } \\
\text { ergometer, \& } \\
\text { iRED }\end{array}$ & $\begin{array}{l}\text { L1, L2, ES, } \\
\text { MF, PS, QL }\end{array}$ & $\begin{array}{l}\text { In-flight exercise helped } \\
\text { prevent paraspinal muscle } \\
\text { mass loss }\end{array}$ \\
\hline $\begin{array}{l}\text { (Koryak } \\
\text { 2019) }\end{array}$ & $\begin{array}{l}\text { Various ISS } \\
\text { missions, } \\
115-380 \text { days }\end{array}$ & $\begin{array}{l}\mathrm{N}=8 \\
\mathrm{M}\end{array}$ & $\begin{array}{l}\text { Architectural and } \\
\text { contractile } \\
\text { properties were } \\
\text { measured using } \\
\text { the tendometry } \\
\text { method. }\end{array}$ & $\begin{array}{l}\text { A four-day } \\
\text { cycle exercise } \\
\text { regimen. }\end{array}$ & TS muscle & $\begin{array}{l}\text { Exercise was not able to } \\
\text { prevent muscle atrophy or } \\
\text { weakness in TS muscles }\end{array}$ \\
\hline $\begin{array}{l}\text { (McNamara } \\
\text { et al. 2019) }\end{array}$ & $\begin{array}{l}\text { Various ISS } \\
\text { missions, } \\
120-180 \text { days }\end{array}$ & $\begin{array}{l}\mathrm{N}=16 \\
15 \mathrm{M}, 1 \mathrm{~F}\end{array}$ & $\begin{array}{l}\text { Muscle volume } \\
\text { and attenuation } \\
\text { were determined } \\
\text { using Helical CT } \\
\text { images }\end{array}$ & $\begin{array}{l}6 \text { subjects kept } \\
\text { in-flight } \\
\text { workout logs. } \\
\text { The subjects } \\
\text { had access to a } \\
\text { CEVIS, TVIS, } \\
\text { \& iRED. }\end{array}$ & $\begin{array}{l}\text { PS, QL, } \\
\text { paraspinal } \\
\text { muscle } \\
\text { groups }\end{array}$ & $\begin{array}{l}\text { Degradation in the QL \& } \\
\text { paraspinal muscles } \\
\text { occurred despite in-flight } \\
\text { exercise }\end{array}$ \\
\hline $\begin{array}{l}\text { (McNamara } \\
\text { et al. 2019) }\end{array}$ & $\begin{array}{l}\text { Various ISS } \\
\text { missions, } \\
120-180 \text { days }\end{array}$ & $\mathrm{N}=6$ & $\begin{array}{l}\text { MRI was used to } \\
\text { measure CSA and } \\
\text { fatty infiltration. }\end{array}$ & $\begin{array}{l}4 \text { subjects kept } \\
\text { in-flight } \\
\text { exercise logs. } \\
\text { The subjects } \\
\text { had access to a } \\
\text { CEVIS, TVIS, } \\
\text { \& ARED. }\end{array}$ & $\begin{array}{l}\text { Neck muscles } \\
\text { at the C1-C2, } \\
\text { C4-C5, C7- } \\
\text { T1, \& T1-T2 } \\
\text { intervertebral } \\
\text { disc levels }\end{array}$ & $\begin{array}{l}\text { In-flight exercise helped } \\
\text { prevent neck musculature } \\
\text { atrophy }\end{array}$ \\
\hline
\end{tabular}


Table 4. Skeletal muscle responses to microgravity in animals - Study characteristics

\begin{tabular}{|c|c|c|c|c|c|c|}
\hline $\begin{array}{l}\text { Author, } \\
\text { Year }\end{array}$ & Mission & Subjects & Study Design & Test \& Method & $\begin{array}{l}\text { Anatomic } \\
\text { site }\end{array}$ & Main Outcomes \\
\hline $\begin{array}{l}\text { (Martin et al. } \\
\text { 1988) }\end{array}$ & $\begin{array}{l}\text { SL-3, } \\
7 \text { days }\end{array}$ & $\begin{array}{l}\mathrm{N}=12 \text { rats, } \\
\mathrm{M}\end{array}$ & $\begin{array}{l}\text { SF group: } \\
n=6 \\
\text { GC group: } \\
n=6\end{array}$ & $\begin{array}{l}\text { Muscle weight, fiber } \\
\text { CSA and } \\
\text { composition, } \\
\text { ATPase, SDH, and } \\
\text { GPD activities were } \\
\text { measured using } \\
\text { histochemical } \\
\text { analysis. }\end{array}$ & $\begin{array}{l}\text { SOL, AL, } \\
\text { PL, EDL, \& } \\
\text { GM muscles }\end{array}$ & $\begin{array}{l}\text { The ability of muscles } \\
\text { to maintain proteins } \\
\text { decreased rapidly }\end{array}$ \\
\hline $\begin{array}{l}\text { (Miu et al. } \\
\text { 1990) }\end{array}$ & $\begin{array}{l}\text { Cosmos } \\
1887 \\
12.5 \text { days }\end{array}$ & $\begin{array}{l}\mathrm{N}=10 \text { rats, } \\
\mathrm{M}\end{array}$ & $\begin{array}{l}\text { SF group: } \\
n=5 \\
\text { GC group: } \\
n=5\end{array}$ & $\begin{array}{l}\text { Histochemistry was } \\
\text { used to measure } \\
\text { fibers atrophy, SDH } \\
\text { and GPD activities }\end{array}$ & $\begin{array}{l}\text { Muscle fibers } \\
\text { of SOL \& } \\
\text { GM }\end{array}$ & $\begin{array}{l}\text { Enzymatic \& cell } \\
\text { volume changes depend } \\
\text { on muscle type, region } \\
\text { of muscle, \& fiber } \\
\text { ATPase type }\end{array}$ \\
\hline $\begin{array}{l}\text { (Musacchia } \\
\text { et al. 1990) }\end{array}$ & $\begin{array}{l}\text { Mission: } \\
\text { unclear, } \\
7 \text { days }\end{array}$ & $\begin{array}{l}\mathrm{N}=42 \text { rats, } \\
\mathrm{M}\end{array}$ & $\begin{array}{l}\text { SF }: n=7 \\
\text { SF VIV: } \\
n=7\end{array}$ & $\begin{array}{l}\text { Muscle mass, CSA, } \\
\text { fiber density, and } \\
\text { capillary density of } \\
\text { the SOL and EDL } \\
\text { were measured. }\end{array}$ & SOL \& EDL & $\begin{array}{l}\text { Muscle mass \& CSA } \\
\text { decreased while fiber } \\
\text { density \& capillary } \\
\text { density increased }\end{array}$ \\
\hline $\begin{array}{l}\text { (Holy and } \\
\text { Mounier } \\
\text { 1991) }\end{array}$ & $\begin{array}{l}\text { Cosmos } \\
\text { 1514: } 5 \\
\text { days, } \\
\text { Cosmos } \\
\text { 1667: } 7 \\
\text { days, }\end{array}$ & $\begin{array}{l}\mathrm{N}=\text { unclear } \\
\text { rats }\end{array}$ & $\begin{array}{l}\text { SF 1514: } \\
n=5 \\
\text { SF } 1667: \\
n=7 \\
\text { SYNC: } \\
n=\text { unclear }\end{array}$ & $\begin{array}{l}\text { Fiber contractile } \\
\text { characteristics were } \\
\text { measured with an } \\
\text { isometric transducer. }\end{array}$ & $\begin{array}{l}\text { SOL, LG, \& } \\
\text { PL muscles }\end{array}$ & $\begin{array}{l}\text { Muscle tension \& } \\
\text { diameter of muscle } \\
\text { fibers were negatively } \\
\text { affected }\end{array}$ \\
\hline $\begin{array}{l}\text { (Bodine- } \\
\text { Fowler et al. } \\
\text { 1992) }\end{array}$ & $\begin{array}{l}\text { Cosmos } \\
2044, \\
14 \text { days }\end{array}$ & $\begin{array}{l}\mathrm{N}=6 \\
\text { macaque, } \\
\mathrm{M}\end{array}$ & $\begin{array}{l}\text { SF: } n=2 \\
\text { GC: } n=4\end{array}$ & $\begin{array}{l}\text { Biopsies were used } \\
\text { to determine fiber } \\
\text { size \& SDH activity. }\end{array}$ & $\begin{array}{l}\text { SOL, TA, \& } \\
\text { GM muscles }\end{array}$ & $\begin{array}{l}\text { SOL \& TA muscle } \\
\text { fibers were not very } \\
\text { affected }\end{array}$ \\
\hline $\begin{array}{l}\text { (Ohira et al. } \\
\text { 1992) }\end{array}$ & $\begin{array}{l}\text { Cosmos } \\
2044 \\
14 \text { days }\end{array}$ & $\begin{array}{l}\mathrm{N}=15 \text { rats, } \\
\mathrm{M}\end{array}$ & $\begin{array}{l}\text { SF: } n=5 \\
\text { HLS: } n=5 \\
\text { GC: } n=5\end{array}$ & $\begin{array}{l}\text { The enzymes \& } \\
\text { morphology } \\
\text { of muscle fibers } \\
\text { were measured using } \\
\text { immunohistochemist } \\
\text { ry. }\end{array}$ & SOL muscle & $\begin{array}{l}\text { CSA reduced more } \\
\text { significantly in slow- } \\
\text { twitch than in fast- } \\
\text { twitch fibers }\end{array}$ \\
\hline $\begin{array}{l}\text { (Bell et al. } \\
\text { 1992) }\end{array}$ & $\begin{array}{l}\text { Cosmos } \\
1887 \\
12.5 \text { days }\end{array}$ & $\begin{array}{l}\mathrm{N}=10 \text { rats, } \\
\mathrm{M}\end{array}$ & $\begin{array}{l}\text { SF: } n=5 \\
\text { GC: } n=5\end{array}$ & $\begin{array}{l}\text { Biopsy and } \\
\text { histochemical } \\
\text { analysis were } \\
\text { utilized to determine } \\
\text { the SDH of FOG \& } \\
\text { SO fibers. }\end{array}$ & SOL muscle & $\begin{array}{l}\text { Effect on muscle fiber } \\
\text { oxidative enzymes } \\
\text { depends on location of } \\
\text { enzyme within fiber }\end{array}$ \\
\hline $\begin{array}{l}\text { (Thomason et } \\
\text { al. 1992) }\end{array}$ & $\begin{array}{l}\text { Cosmos } \\
2044, \\
14 \text { days }\end{array}$ & $\mathrm{N}=$ unclear & $\begin{array}{l}\text { SF: unclear } \\
\text { SYNC: unclear }\end{array}$ & $\begin{array}{l}\text { Contractile protein } \\
\text { gene expression was } \\
\text { measured. }\end{array}$ & unclear & $\begin{array}{l}\text { Pretranslational } \\
\text { regulation of contractile } \\
\text { protein gene expression } \\
\text { of skeletal muscle } \\
\text { changed }\end{array}$ \\
\hline $\begin{array}{l}\text { (Baldwin et } \\
\text { al. 1993) }\end{array}$ & $\begin{array}{l}\text { SLS-1, } \\
9 \text { days }\end{array}$ & $\begin{array}{l}\mathrm{N}=40 \text { rats, } \\
\mathrm{M}\end{array}$ & $\begin{array}{l}\text { SF: } n=20 \\
\text { CG: } n=20\end{array}$ & $\begin{array}{l}\text { Body weight, muscle } \\
\text { weight, protein } \\
\text { substrate oxidation, } \\
\& \text { oxidative enzymes } \\
\text { were assessed. }\end{array}$ & $\begin{array}{l}\text { VI, VL, TA } \\
\text { muscles }\end{array}$ & $\begin{array}{l}\text { VI and VL muscle } \\
\text { weights were reduced }\end{array}$ \\
\hline $\begin{array}{l}\text { (Henriksen et } \\
\text { al. 1993) }\end{array}$ & $\begin{array}{l}\text { Discovery, } \\
4.5 \text { days }\end{array}$ & $\begin{array}{l}\mathrm{N}=16 \\
\text { rats, } \\
\mathrm{F}\end{array}$ & $\begin{array}{l}\text { SF SOL: } \mathrm{n}=8 \\
\text { CON SOL: } \mathrm{n}=8 \\
\text { SF EDL: } \mathrm{n}=8 \\
\text { CON EDL: } \mathrm{n}=8\end{array}$ & $\begin{array}{l}\text { Muscle weight and } \\
\text { interstitial fluid } \\
\text { volume were tested. }\end{array}$ & SOL, EDL & $\begin{array}{l}\text { Soleus weight decreased } \\
\text { while soleus interstitial } \\
\text { fluid volume increased }\end{array}$ \\
\hline $\begin{array}{l}\text { (Haddad et } \\
\text { al. 1993) }\end{array}$ & $\begin{array}{l}\text { SLS-1, } \\
9 \text { days }\end{array}$ & $\begin{array}{l}\mathrm{N}=20 \\
\text { Rats, } \mathrm{M}\end{array}$ & $\begin{array}{l}\text { SF: } n=10 \\
\text { GC: } n=10\end{array}$ & $\begin{array}{l}\text { MHC expression } \\
\text { was measured. }\end{array}$ & VI, VL & $\begin{array}{l}\text { VI atrophied } \\
\text { significantly while the } \\
\text { tibialis anterior (TA) } \\
\text { and VL did not }\end{array}$ \\
\hline
\end{tabular}


Table 4. (continued)

\begin{tabular}{|c|c|c|c|c|c|c|}
\hline $\begin{array}{l}\text { (Caiozzo et } \\
\text { al. 1994) }\end{array}$ & $\begin{array}{l}\text { STS-54, } 6 \\
\text { days }\end{array}$ & $\begin{array}{l}\mathrm{N}=12 \\
\text { rats, } \\
\mathrm{M}\end{array}$ & $\begin{array}{l}\text { SF: } n=6 \\
\text { GC: } n=6\end{array}$ & $\begin{array}{l}\text { Contractile, } \\
\text { biochemical, \& } \\
\text { histochemical } \\
\text { properties were } \\
\text { assessed. }\end{array}$ & SOL & $\begin{array}{l}\text { SOL muscle became } \\
\text { both weaker and faster }\end{array}$ \\
\hline $\begin{array}{l}\text { (Esser and } \\
\text { Hardeman } \\
\text { 1995) }\end{array}$ & $\begin{array}{l}\text { SLS-1, } \\
9 \text { days }\end{array}$ & $\begin{array}{l}\mathrm{N}=20 \\
\text { rats, } \\
\mathrm{M}\end{array}$ & $\begin{array}{l}\text { SF: } n=10 \\
\text { GC: } n=10\end{array}$ & $\begin{array}{l}\text { Contractile protein } \\
\text { isoform mRNAs in } \\
\text { fast and slow muscle } \\
\text { fibers were } \\
\text { investigated. }\end{array}$ & SOL, EDL & $\begin{array}{l}\text { Fast mRNA levels } \\
\text { increased in six } \\
\text { contractile protein gene } \\
\text { families }\end{array}$ \\
\hline $\begin{array}{l}\text { (Allen et al. } \\
\text { 1996) }\end{array}$ & $\begin{array}{l}\text { SLS-2, } \\
14 \text { days }\end{array}$ & $\begin{array}{l}\mathrm{N}=5 \\
\text { rats, } \\
\mathrm{M}\end{array}$ & $\begin{array}{l}\text { SF: } n=5 \\
\text { GC: } n=\text { unclear }\end{array}$ & $\begin{array}{l}\text { Myonuclear number, } \\
\text { morphology, \& } \\
\text { MHC composition } \\
\text { were tested. }\end{array}$ & SOL & $\begin{array}{l}\text { SOL myonuclear } \\
\text { number \& size of fibers } \\
\text { expressing type I MHC } \\
\text { decreased }\end{array}$ \\
\hline $\begin{array}{l}\text { (Riley et al. } \\
\text { 1996) }\end{array}$ & $\begin{array}{l}\text { SLS-1: } \\
9 \text { days, } \\
\text { SLS-2: } \\
14 \text { days }\end{array}$ & $\begin{array}{l}\mathrm{N}-51 \\
\text { rats, } \\
\mathrm{M}\end{array}$ & $\begin{array}{l}\text { SLS- } 1: \mathrm{n}=15, \\
\text { SLS- } 2: \mathrm{n}=11, \\
\text { GC SLS-1: } \mathrm{n}=15 \text {, } \\
\text { GC SLS- } 2: \mathrm{n}=10\end{array}$ & $\begin{array}{l}\text { Myonuclear number, } \\
\text { fiber size, \& MHC } \\
\text { expression }\end{array}$ & $\begin{array}{l}\text { AL, EDL, } \\
\text { SOL }\end{array}$ & $\begin{array}{l}\text { SOL, AL, \& EDL of } \\
\text { rats were negatively } \\
\text { impacted by SF }\end{array}$ \\
\hline $\begin{array}{l}\text { (Caiozzo et } \\
\text { al. 1996) }\end{array}$ & $\begin{array}{l}\text { STS-58 } \\
14 \text { days }\end{array}$ & $\begin{array}{l}\mathrm{N}=17 \\
\text { rats, } \\
\mathrm{M}\end{array}$ & $\begin{array}{l}S F: n=5 ; C G \\
L+14: n=6, \\
C G L+0: n=6\end{array}$ & $\begin{array}{l}\text { Contractile } \\
\text { properties, MHC } \\
\text { protein, and mRNA } \\
\text { isoform content were } \\
\text { measured. }\end{array}$ & $\begin{array}{l}\text { SOL, VI, } \\
\text { TA, \& PL }\end{array}$ & $\begin{array}{l}\text { Short duration SF } \\
\text { changes muscle mass, } \\
\text { contractile properties, } \\
\text { MHC, and mRNA } \\
\text { isoform contents }\end{array}$ \\
\hline $\begin{array}{l}\text { (Vandenburg } \\
\text { het al. 1999) }\end{array}$ & $\begin{array}{l}\text { STS-77, } \\
10 \text { days }\end{array}$ & $\begin{array}{l}\mathrm{N}=36 \\
\text { bioartifici } \\
\text { al } \\
\text { muscles }\end{array}$ & $\begin{array}{l}\text { SF: } n=18, \\
\text { GC: } n=18\end{array}$ & $\begin{array}{l}\text { Protein turnover, } \\
\text { MHC, \& } \\
\text { morphology of } \\
\text { muscle fibers were } \\
\text { tested. }\end{array}$ & BAM & $\begin{array}{l}\text { Muscle fiber } \\
\text { atrophy occurred \& } \\
\text { protein synthesis rates } \\
\text { decreased without } \\
\text { alterations in protein } \\
\text { degradation }\end{array}$ \\
\hline $\begin{array}{l}\text { (Kraemer et } \\
\text { al. 2000) }\end{array}$ & $\begin{array}{l}\text { PSE-03, } \\
10 \text { days }\end{array}$ & $\begin{array}{l}\mathrm{N}=24 \\
\text { rats, } \\
\mathrm{M}\end{array}$ & $\begin{array}{l}\text { SF: } n=12, \\
\text { GC: } n=12\end{array}$ & $\begin{array}{l}\text { CSA \& MHC were } \\
\text { investigated. }\end{array}$ & EDL, GAS & $\begin{array}{l}\text { EDL and GAS muscles } \\
\text { exposed to short-term } \\
\mu G \text { show rapid } \\
\text { plasticity }\end{array}$ \\
\hline $\begin{array}{l}\text { (Lalani et al. } \\
\text { 2000) }\end{array}$ & $\begin{array}{l}\text { STS-90, } \\
17 \text { days }\end{array}$ & $\begin{array}{l}\mathrm{N}=36 \\
\text { rats, } \\
\mathrm{M}\end{array}$ & $\begin{array}{l}\text { SF R1: } n=6 \\
\text { SF R13: } n=6 \\
\text { GC VIV: } n=12 \\
\text { VIV R3: } n=6 \\
\text { VIV R15: } n=6 \\
\text { GC flight: } n=12 \\
\text { R5: } n=6 \\
\text { R17: } n=6\end{array}$ & $\begin{array}{l}\text { Levels of myostatin, } \\
\text { IGF-I and -II, \& } \\
\text { muscle proteolysis \& } \\
\text { apoptosis were } \\
\text { investigated. }\end{array}$ & $\begin{array}{l}\text { TA, biceps } \\
\text { femoris, } \\
\text { Quad, and } \\
\text { GAS } \\
\text { muscles. }\end{array}$ & $\begin{array}{l}\text { Intramuscular } \\
\text { myostatin mRNA } \\
\text { concentrations increase } \\
\text { \& IGF-II mRNA } \\
\text { concentrations } \\
\text { decreased }\end{array}$ \\
\hline $\begin{array}{l}\text { (Shenkman } \\
\text { et al. 2003) }\end{array}$ & $\begin{array}{l}\text { BION-11, } \\
14 \text { days }\end{array}$ & $\begin{array}{l}\mathrm{N}=10 \\
\text { monkeys }\end{array}$ & $\begin{array}{l}\text { SF }: n=2 \\
C C: n=3 \\
\text { HC: } n=5\end{array}$ & $\begin{array}{l}\text { Biopsy was used to } \\
\text { measure } \\
\text { fiber sizes, protein } \\
\text { level, number of } \\
\text { capillaries, and SDH } \\
\text { activity. }\end{array}$ & SOL & $\begin{array}{l}\text { Fiber sizes, number of } \\
\text { capillaries, \& SDH } \\
\text { activity decreased }\end{array}$ \\
\hline $\begin{array}{l}\text { (Hansen et } \\
\text { al. 2004) }\end{array}$ & $\begin{array}{l}\text { SLS-2, } \\
14 \text { days }\end{array}$ & $\begin{array}{l}\mathrm{N}=10 \\
\text { rats, } \\
\mathrm{M}\end{array}$ & $\begin{array}{l}\text { SF: } n=5 \\
\text { GC: } n=5\end{array}$ & $\begin{array}{l}\text { Fiber type, fiber size, } \\
\text { CSA, MHC isoform } \\
\text { analysis, SDH } \\
\text { activity in single } \\
\text { fibers }\end{array}$ & DIA & $\begin{array}{l}\text { Structural or metabolic } \\
\text { factors of DIA were not } \\
\text { greatly affected }\end{array}$ \\
\hline $\begin{array}{l}\text { (Schuenke } \\
\text { et al. 2009) }\end{array}$ & $\begin{array}{l}\text { STS-62, } \\
14 \text { days }\end{array}$ & $\begin{array}{l}\mathrm{N}=24 \\
\text { rats, } \\
\mathrm{F}\end{array}$ & $\begin{array}{l}\text { SF: } n=12 \\
\text { GC: } n=12\end{array}$ & $\begin{array}{l}\text { CSA, muscle mass, } \\
\text { MHC isoform } \\
\text { content were } \\
\text { assessed. }\end{array}$ & $\begin{array}{l}\text { Hindlimb } \\
\text { and DIA }\end{array}$ & $\begin{array}{l}\text { Microgravity has } \\
\text { a greater negative effect } \\
\text { on hindlimb muscles } \\
\text { than on DIA }\end{array}$ \\
\hline
\end{tabular}


Table 4. (continued)

\begin{tabular}{|c|c|c|c|c|c|c|}
\hline $\begin{array}{l}\text { (Sandona } \\
\text { et al. 2012) }\end{array}$ & $\begin{array}{l}\text { Discovery, } \\
91 \text { days }\end{array}$ & $\begin{array}{l}\mathrm{N}=7 \\
\text { mice, } \\
\mathrm{M}\end{array}$ & $\begin{array}{l}\text { SF MDS: } n=1 \\
\text { GC: } n=3 \\
\text { GC MDS: } n=3\end{array}$ & $\begin{array}{l}\text { CSA of muscle fibers } \\
\text { were measured. }\end{array}$ & SOL \& EDL & $\begin{array}{l}\text { SOL shows greater } \\
\text { atrophy than EDL }\end{array}$ \\
\hline $\begin{array}{l}\text { (Gambara } \\
\text { et al. 2017) }\end{array}$ & $\begin{array}{l}\text { Bion-M1, } \\
30 \text { days }\end{array}$ & $\begin{array}{l}\mathrm{N}=15 \\
\text { mice, } \\
\mathrm{M}\end{array}$ & $\begin{array}{l}\text { SF: } n=5 \\
\text { GC: } n=5 \\
\text { FC: } n=5\end{array}$ & $\begin{array}{l}\text { Gene expression in } \\
\text { calf muscle fibers } \\
\text { were tested using } \\
\text { microarray } \\
\text { technology. }\end{array}$ & SOL & $\begin{array}{l}\text { Gene expression was } \\
\text { greatly affected in SOL } \\
\text { compared to EDL }\end{array}$ \\
\hline $\begin{array}{l}\text { (Tascher } \\
\text { et al. 2017) }\end{array}$ & $\begin{array}{l}\text { Bion-M1, } \\
30 \text { days }\end{array}$ & $\begin{array}{l}\mathrm{N}=24 \\
\text { mice, } \\
\mathrm{M}\end{array}$ & $\begin{array}{l}\text { SF: } n=5 \\
\text { Rec: } n=4 \\
\text { GC: } n=5 \\
\text { VC: } n=5 \\
\text { RC: } n=5\end{array}$ & $\begin{array}{l}\text { Muscle mass, fiber } \\
\text { type, proteome } \\
\text { changes, protein } \\
\text { balance, metabolism, } \\
\text { mitochondrial } \\
\text { function, antioxidant } \\
\text { proteins }\end{array}$ & $\begin{array}{l}\text { VL, EDL, } \\
\text { SOL }\end{array}$ & $\begin{array}{l}\text { Changes in protein } \\
\text { balance, mitochondrial } \\
\text { function, metabolism, } \\
\text { structure, fiber types, } \\
\text { calcium signaling, \& } \\
\text { telomere maintenance } \\
\text { led to muscle size \& } \\
\text { performance decreases }\end{array}$ \\
\hline $\begin{array}{l}\text { (Radugina } \\
\text { et al. 2018) }\end{array}$ & $\begin{array}{l}\text { Bion-M1, } \\
30 \text { days }\end{array}$ & $\begin{array}{l}\mathrm{N}=27 \\
\text { mice, } \\
\mathrm{M}\end{array}$ & $\begin{array}{l}\text { SF 1: } n=6 \\
\text { ASYNC CG: } \\
n=7 \\
\text { Viv CG: } n=14\end{array}$ & $\begin{array}{l}\text { Muscle fiber atrophy, } \\
\text { myofibril degradation, } \\
\text { \& regenerative } \\
\text { hypoplasia }\end{array}$ & Femoral Quad & $\begin{array}{l}\text { Reductions in myofiber } \\
\text { quantity and impairment } \\
\text { of regeneration occurred }\end{array}$ \\
\hline
\end{tabular}

Table 5. Adaptation of the skeletal system to microgravity in humans - Study characteristics

\begin{tabular}{|c|c|c|c|c|c|c|}
\hline Author, Year & Mission & Subjects & $\begin{array}{l}\text { Exercise } \\
\text { Regimen }\end{array}$ & $\begin{array}{l}\text { Test \& } \\
\text { Methods }\end{array}$ & Anatomic site & Main Outcomes \\
\hline $\begin{array}{l}\text { (Mack et al. } \\
\text { 1967) }\end{array}$ & $\begin{array}{l}\text { Gemini 4: } 4 \\
\text { days, } \\
\text { Gemini 5: } 8 \\
\text { days, } \\
\text { Gemini 7: } \\
\text { 14 days }\end{array}$ & $\begin{array}{l}N=6 \\
G 4: n=2 \text { G5: } \\
n=2 \text { G7: } n=2\end{array}$ & $\begin{array}{l}\text { A planned } \\
\text { exercise program } \\
\text { was used for the } \\
\text { first time in } \\
\text { Gemini VII }\end{array}$ & BMD, x-rays & $\begin{array}{l}\text { CAL, talus, } \\
\text { phalanges, \& } \\
\text { capitate }\end{array}$ & $\begin{array}{l}\text { Length of SF and other } \\
\text { variables determined } \\
\text { amount of bone loss }\end{array}$ \\
\hline (Whedon 1971) & $\begin{array}{l}\text { Gemini 7: } \\
14 \text { days }\end{array}$ & $\mathrm{N}=2$ & Unstated & Urinary analysis & Total body & $\begin{array}{l}\text { Urinary calcium levels } \\
\text { increased }\end{array}$ \\
\hline $\begin{array}{l}\text { (Mack and Vogt } \\
\text { 1971) }\end{array}$ & $\begin{array}{l}\text { Apollo 7: } 11 \\
\text { days, } \\
\text { Apollo 8: } 6 \\
\text { days }\end{array}$ & $\begin{array}{l}\mathrm{N}=6 \\
\text { Apollo 7: } \mathrm{n}=3 \\
\text { Apollo 8: } \mathrm{n}=3\end{array}$ & $\begin{array}{l}\text { Isotonic \& } \\
\text { isometric exercise } \\
\text { in a recumbent } \\
\text { position. }\end{array}$ & $\mathrm{x}$ - rays & $\begin{array}{l}\text { Distal ulna, } \\
\text { phalanges, wrist, } \\
\text { CAL, \& talus }\end{array}$ & $\begin{array}{l}\text { Loss of calcium and } \\
\text { bone density were } \\
\text { influenced by dietary } \\
\text { intake of calcium \& } \\
\text { exercise }\end{array}$ \\
\hline (Vogel 1975) & $\begin{array}{l}\text { Apollo 14, } \\
15, \& 16: \\
\text { various days }\end{array}$ & $\begin{array}{l}\mathrm{N}=27 \\
\text { SF: } n=9 \\
\text { BR CON: } \\
n=15 \text { SMEAT } \\
\text { CON: } n=3\end{array}$ & Unstated & $\begin{array}{l}\text { Bone mineral } \\
\text { changes using } \\
\text { x-rays }\end{array}$ & $\begin{array}{l}\text { CAL, RAD \& } \\
\text { ulna }\end{array}$ & $\begin{array}{l}\text { Trabecular \& weight- } \\
\text { bearing bones are more } \\
\text { susceptible to the } \\
\text { negative effects of SF }\end{array}$ \\
\hline (Smith 1977) & $\begin{array}{l}\text { Skylab 2,3, } \\
4: 28-84 \\
\text { days }\end{array}$ & $\begin{array}{l}\mathrm{N}=22 \\
\text { Flight: } \mathrm{n}=9 \\
\text { Control: } \mathrm{n}=13\end{array}$ & Unstated & $\begin{array}{l}\text { BMC using } \\
\text { photon } \\
\text { absorptiometry }\end{array}$ & $\begin{array}{l}\text { CAL \& RAD \& } \\
\text { ulna }\end{array}$ & $\begin{array}{l}\text { Os calcis mineral losses } \\
\text { varied among individuals }\end{array}$ \\
\hline $\begin{array}{l}\text { (Rambaut and } \\
\text { Johnston 1979) }\end{array}$ & $\begin{array}{l}\text { Skylab 2,3, } \\
4 ; 28-84 \\
\text { days }\end{array}$ & $\mathrm{N}=$ unclear & Unstated & $\begin{array}{l}\text { Body calcium, } \\
\text { BMD, measured } \\
\text { by urinary, \& } \\
\text { fecal analysis }\end{array}$ & $\begin{array}{l}\text { CAL, whole } \\
\text { body }\end{array}$ & $\begin{array}{l}\text { Urinary calcium began } \\
\text { to increase almost } \\
\text { immediately }\end{array}$ \\
\hline $\begin{array}{l}\text { (Oganov et al. } \\
\text { 1991) }\end{array}$ & $\begin{array}{l}\text { Saljut- } 7 \\
150,211 \\
237 \text { days }\end{array}$ & $\mathrm{N}=7$ & $\begin{array}{l}\text { The exercise } \\
\text { regimen is not } \\
\text { described in detail }\end{array}$ & $\begin{array}{l}\text { BMD was } \\
\text { determined } \\
\text { using QCT } \\
\text { analysis }\end{array}$ & $\begin{array}{l}\text { Lumbar } \\
\text { vertebral, \& back } \\
\text { muscles }\end{array}$ & $\begin{array}{l}\text { Volume and density of } \\
\text { back muscles slightly } \\
\text { decreased due to } \\
\text { exercise } \\
\text { countermeasures }\end{array}$ \\
\hline
\end{tabular}


Table 5. (continued)

\begin{tabular}{|c|c|c|c|c|c|c|}
\hline $\begin{array}{l}\text { (Collet et al. } \\
\text { 1997) }\end{array}$ & $\begin{array}{l}\text { EuroMir } 94, \\
\text { 30-180 days }\end{array}$ & $\mathrm{N}=2$ & Unstated & $\begin{array}{l}\text { BMD and bone } \\
\text { metabolism } \\
\text { using QCT, } \\
\text { ultrasound \& } \\
\text { bone markers }\end{array}$ & Tibia, \& RAD & $\begin{array}{l}\text { Longer duration SF lead } \\
\text { to greater negative } \\
\text { changes in BMD \& } \\
\text { bone metabolism }\end{array}$ \\
\hline (Smith 1999) & $\begin{array}{l}\text { MIR 18, } \\
115 \text { days }\end{array}$ & $\mathrm{N}=3$ & Unstated & $\begin{array}{l}\text { Calcium } \\
\text { analysis using } \\
\text { urinary samples } \\
\& \text { biomarkers }\end{array}$ & Whole body & $\begin{array}{l}\text { Urinary calcium } \\
\text { excretion and bone } \\
\text { resorption increased and } \\
\text { recovered slowly }\end{array}$ \\
\hline $\begin{array}{l}\text { (Vico et al. } \\
2000)\end{array}$ & $\begin{array}{l}\text { MIR } \\
\text { 30-180 days }\end{array}$ & $\mathrm{N}=15$ & Unstated & $\begin{array}{l}\text { BMD was } \\
\text { measured by } \\
\text { pQCT system }\end{array}$ & $\begin{array}{l}\text { Distal RAD \& } \\
\text { tibia }\end{array}$ & $\begin{array}{l}\text { Greater bone loss } \\
\text { occurred in the } \\
\text { trabecular bone than in } \\
\text { the cortex }\end{array}$ \\
\hline $\begin{array}{l}\text { (Lang et al. } \\
\text { 2004) }\end{array}$ & $\begin{array}{l}\text { ISS 2-6, } \\
120-180 \\
\text { days }\end{array}$ & $\mathrm{N}=14$ & Unstated & $\begin{array}{l}\text { DXA, vQCT \& } \\
\text { QUS }\end{array}$ & $\begin{array}{l}\text { Hip, lumbar } \\
\text { spine, heel }\end{array}$ & $\begin{array}{l}\text { Greater bone losses } \\
\text { occurred in the hip } \\
\text { compared to bone losses } \\
\text { in the spine }\end{array}$ \\
\hline $\begin{array}{l}\text { (Smith et al. } \\
\text { 2005) }\end{array}$ & $\begin{array}{l}\text { Shuttle-Mir } \\
\text { Science, } \\
120-180 \\
\text { days }\end{array}$ & $\mathrm{N}=13$ & Unstated & $\begin{array}{l}\text { Blood \& urine } \\
\text { analysis to } \\
\text { determine bone } \\
\& \text { calcium } \\
\text { metabolism }\end{array}$ & $\begin{array}{l}\text { Total bone \& } \\
\text { calcium markers }\end{array}$ & $\begin{array}{l}\text { Bone loss is caused by } \\
\text { high bone resorption and } \\
\text { low intestinal calcium } \\
\text { absorption }\end{array}$ \\
\hline $\begin{array}{l}\text { (Keyak et al. } \\
\text { 2009) }\end{array}$ & $\begin{array}{l}\text { ISS, } \\
135-195 \\
\text { days }\end{array}$ & $\mathrm{N}=13$ & $\begin{array}{l}\text { Customized } \\
\text { exercise regimen. } \\
2 \mathrm{~h} \text { /day, } 4 \\
\text { days/week. Using } \\
\text { a treadmill, cycle, } \\
\& \text { iRED }\end{array}$ & $\begin{array}{l}\text { Bone strength } \\
\text { was measured } \\
\text { using QCT scan }\end{array}$ & Proximal FEM & $\begin{array}{l}\text { Reductions of proximal } \\
\text { femoral strength could } \\
\text { lead to an enhanced risk } \\
\text { for hip fractures }\end{array}$ \\
\hline $\begin{array}{l}\text { (Vico et al. } \\
\text { 2017) }\end{array}$ & $\begin{array}{l}\text { ISS }(2007- \\
2013) \\
120-180 \\
\text { days }\end{array}$ & $\mathrm{N}=13$ & Unstated & $\begin{array}{l}\text { QCT and blood } \\
\text { sample analysis }\end{array}$ & $\begin{array}{l}\text { Lumbar spine, } \\
\text { femoral neck, } \\
\text { total hip, distal } \\
\text { RAD \& tibia }\end{array}$ & $\begin{array}{l}\text { Certain changes could be } \\
\text { partly irreversible }\end{array}$ \\
\hline
\end{tabular}

Note: All the studies in this section have a pre-posttest design, with or without control arms.

Table 6. Adaptation of the skeletal system to microgravity in animals - Study Characteristics

\begin{tabular}{|c|c|c|c|c|c|c|}
\hline $\begin{array}{l}\text { Author, } \\
\text { year }\end{array}$ & $\begin{array}{l}\text { Mission, } \\
\text { Duration }\end{array}$ & Subjects & Study design & Test \& Method & Anatomic site & Main Outcomes \\
\hline (Morey 1978) & $\begin{array}{l}\text { Cosmos } 782- \\
\text { Bion 3, } \\
19.5 \text { days }\end{array}$ & $\begin{array}{l}\mathrm{N}=63 \\
\text { Rats }\end{array}$ & $\begin{array}{l}\text { SF: } n=6, S F \\
\text { CON: } n=6 \\
\text { GC: } n=6 \\
\text { Post-flight: SF: } \\
n=6, \text { SF CON: } \\
n=6, \text { GC: } n=6\end{array}$ & $\begin{array}{l}\text { Tetracycline injection } \\
\text { was used as a } \\
\text { fluorescent label }\end{array}$ & $\begin{array}{l}\text { Tibia, } \\
\text { tibiofibular } \\
\text { junction }\end{array}$ & $\begin{array}{l}\text { Bone growth completely } \\
\text { stopped in-flight }\end{array}$ \\
\hline $\begin{array}{l}\text { (Gurovsky } \\
\text { et al. 1980) }\end{array}$ & $\begin{array}{l}\text { Cosmos- } 93 \\
18.5 \text { days }\end{array}$ & $\begin{array}{l}\mathrm{N}=30 \\
\text { Rats, } \\
\mathrm{M}\end{array}$ & $\begin{array}{l}\text { SF: } n=20, \\
\text { Centrifuge: } n=10 \\
\text { Control: } \\
n=\text { unstatted }\end{array}$ & $\begin{array}{l}\text { Bone and muscle } \\
\text { analysis of whole body }\end{array}$ & $\begin{array}{l}\text { Whole body, } \\
\text { FEM, tibia, \& } \\
\text { SOL }\end{array}$ & $\begin{array}{l}\text { Weight gain delay, } \\
\text { osteoporosis, bone density } \\
\text { loss, mineral content loss, } \\
\& \text { decreased bending } \\
\text { strength in the femoral \& } \\
\text { tibial bone }\end{array}$ \\
\hline $\begin{array}{l}\text { (Cann and } \\
\text { Adachi } \\
\text { 1983) }\end{array}$ & $\begin{array}{l}\text { Cosmos } \\
1129 \\
10 \text { days }\end{array}$ & $\begin{array}{l}\mathrm{N}=10 \\
\text { Rats }\end{array}$ & $\begin{array}{l}\text { SF: } n=5 \text { SYNC } \\
\text { CON: } n=5\end{array}$ & $\begin{array}{l}\text { Continuous tracer } \\
\text { techniques for bone } \\
\text { resorption analysis }\end{array}$ & Whole body & $\begin{array}{l}\text { Mineral homeostasis did } \\
\text { not adapt to SF conditions }\end{array}$ \\
\hline
\end{tabular}


Table 6. (continued)

\begin{tabular}{|c|c|c|c|c|c|c|}
\hline $\begin{array}{l}\text { (Jee et al. } \\
\text { 1983) }\end{array}$ & $\begin{array}{l}\text { COSMOS } \\
1129 \\
18.5 \text { days }\end{array}$ & $\begin{array}{l}\mathrm{N}=\text { uncle } \\
\text { ar } \\
\text { Rats, } \\
\mathrm{M}\end{array}$ & $\begin{array}{l}\text { SF: } n=\text { Unclear; } \\
\text { GC: } n=\text { Unclear }\end{array}$ & Bone formation & $\begin{array}{l}\text { Proximal } \\
\text { humeral } \\
\text { metaphysis }\end{array}$ & $\begin{array}{l}\text { Decreased number of } \\
\text { osteoblasts at the growth } \\
\text { cartilage metaphyseal } \\
\text { junction }\end{array}$ \\
\hline $\begin{array}{l}\text { (Jee et al. } \\
\text { 1983) }\end{array}$ & $\begin{array}{l}\text { Cosmos } \\
1667 \\
7 \text { days }\end{array}$ & $\begin{array}{l}\mathrm{N}=14 \\
\text { Rats, } \\
\mathrm{M}\end{array}$ & $\begin{array}{l}\text { SF: } n=7 \\
\text { GC in USSR: } \\
n=7 \\
\text { GC in France: } \\
n=10\end{array}$ & $\begin{array}{l}\text { Histomorphometry } \\
\text { analysis }\end{array}$ & $\begin{array}{l}\text { Tibia \& FEM } \\
\text { metaphysis, } \\
\text { 8th thoracic, \& } \\
\text { 1st lumbar } \\
\text { vertebrae }\end{array}$ & $\begin{array}{l}\text { Osteoid surfaces were } \\
\text { significantly decreased }\end{array}$ \\
\hline $\begin{array}{l}\text { (Mechanic et } \\
\text { al. 1990) }\end{array}$ & $\begin{array}{l}\text { Biosatellite, } \\
12.5 \text { days }\end{array}$ & $\begin{array}{l}\mathrm{N}=40 \\
\text { Rats }\end{array}$ & $\begin{array}{l}\text { Basal: } n=10 \\
\text { SF: } n=10 \\
\text { SYNC control: } \\
n=10 \\
\text { VIV control: } \\
n=10\end{array}$ & $\begin{array}{l}\mu \mathrm{CT}, \mathrm{X} \text {-ray, \& BSE } \\
\text { calcium \& osteocalcin } \\
\text { levels were measured }\end{array}$ & FEM & $\begin{array}{l}\text { Mineralization in the } \\
\text { distal half of the diaphysis } \\
\text { decreased }\end{array}$ \\
\hline $\begin{array}{l}\text { (Zernicke et al. } \\
\text { 1990) }\end{array}$ & $\begin{array}{l}\text { Cosmos } \\
1887 \\
12.5 \text { days }\end{array}$ & $\begin{array}{l}\mathrm{N}=20 \\
\text { Rats, } \\
\mathrm{M}\end{array}$ & $\begin{array}{l}\text { SF: } n=5 \\
\text { Basal control: } \\
n=5 \\
\text { SYNC control: } \\
n=5 \\
\text { Viv controls: } \\
n=5\end{array}$ & $\begin{array}{l}\text { Biomechanical } \\
\text { (Vertebral Compression } \\
\text { Test), \& biochemical } \\
\text { testing }\end{array}$ & $\begin{array}{l}\text { L6 vertebral } \\
\text { body mass }\end{array}$ & $\begin{array}{l}\text { Decreases in strength, } \\
\text { increases in stiffness, and } \\
\text { lower amounts of } \\
\text { hydroxypyridinone } \\
\text { observed }\end{array}$ \\
\hline $\begin{array}{l}\text { (Backup et al. } \\
\text { 1994) }\end{array}$ & $\begin{array}{l}\text { Discovery: } 4 \\
\text { days, } \\
\text { Columbia: } 10 \\
\text { days }\end{array}$ & $\begin{array}{l}\mathrm{N}=26 \\
\text { Rats, } \\
\mathrm{M}\end{array}$ & $\begin{array}{l}\text { SF: } n=8 \& n=6 \\
\text { GC: } n=6 \& n=6\end{array}$ & $\begin{array}{l}\text { Histomorphometry, } \\
\text { osteocalcin \& type I } \\
\text { collagen }\end{array}$ & $\begin{array}{l}\text { FEM \& } \\
\text { humerus, } \\
\text { radius, ulna, } \\
\text { calvaria, } \\
\text { biceps }\end{array}$ & $\begin{array}{l}\text { Decreased expression of } \\
\text { some bone-specific genes } \\
\& \text { decreased bone } \\
\text { formation }\end{array}$ \\
\hline $\begin{array}{l}\text { (Zerath et al. } \\
\text { 1996) }\end{array}$ & $\begin{array}{l}\text { Cosmos } 2229 \\
(\text { Bion } 10- \\
\text { 1992), } 11.5 \\
\text { days }\end{array}$ & $\begin{array}{l}\mathrm{N}=4 \\
\text { Monkey } \\
\mathrm{S} \\
\mathrm{M}\end{array}$ & $\begin{array}{l}\text { SF: } n=2 \\
\text { Mock-up: } n=2 \\
\text { Viv: } n=4 \\
\text { GC chair: } n=4\end{array}$ & $\begin{array}{l}\text { dual-photon } \\
\text { absorptiometry, } \\
\text { histomorphometry, \& } \\
\text { BSE analysis }\end{array}$ & $\begin{array}{l}\text { Iliac trabecular } \\
\text { area }\end{array}$ & $\begin{array}{l}\text { Bone loss in the iliac } \\
\text { trabecular area \& altered } \\
\text { mineralizing activity }\end{array}$ \\
\hline $\begin{array}{l}\text { (Ghosh et al. } \\
\text { 2016) }\end{array}$ & $\begin{array}{l}\text { STS-131: } 15 \\
\text { days } \\
\text { STS-135: } 13 \\
\text { days }\end{array}$ & $\begin{array}{l}\mathrm{N}=28 \\
\text { Mice, } \\
\mathrm{F}\end{array}$ & $\begin{array}{l}\text { SF: } \\
\text { STS-131: } \mathrm{n}=8 \\
\text { STS-135: } \mathrm{n}=5 \\
\text { GC STS-131: } \\
\mathrm{n}=8 \\
\text { GC STS-135: } \\
\mathrm{n}=7\end{array}$ & High-resolution $\mu \mathrm{CT}$ & Mandible bone & $\begin{array}{l}\text { Minerals were } \\
\text { redistributed within the } \\
\text { skeleton }\end{array}$ \\
\hline $\begin{array}{l}\text { (Macaulay } \\
\text { et al. 2017) }\end{array}$ & Bion-M1 & $\begin{array}{l}\mathrm{N}=13 \\
\text { Mice, } \\
\mathrm{M}\end{array}$ & $\begin{array}{l}\text { SF: } n=6 \\
\text { GC: } n=7\end{array}$ & $\begin{array}{l}\text { Bone structure and } \\
\text { histomorphometry were } \\
\text { determined using } \mu \mathrm{CT}\end{array}$ & $\begin{array}{l}\text { Calvariae } \\
\text { bones in the } \\
\text { skull }\end{array}$ & $\begin{array}{l}\text { SF might alter alvaria } \\
\text { mechanotransduction } \\
\text { pathways }\end{array}$ \\
\hline $\begin{array}{l}\text { (Dagdeviren } \\
\text { et al. 2018) }\end{array}$ & $\begin{array}{l}\text { STS-135: } \\
\text { 13 days } \\
\text { Bion-M1: } 30 \\
\text { days }\end{array}$ & $\begin{array}{l}\mathrm{N}=41 \\
\text { Mice, } \\
\text { F \& M }\end{array}$ & $\begin{array}{l}\text { AEMs flight: } \\
\mathrm{n}=7 \\
\text { BOS flight: } \mathrm{n}=6 \\
\text { AEMs GC: } \mathrm{n}=7 \\
\text { BOS GC: } \mathrm{n}=7 \\
\text { AEMs Viv } \\
\text { Control: } \mathrm{n}=7 \\
\text { BOS Viv } \\
\text { Control: } \mathrm{n}=7\end{array}$ & $\begin{array}{l}\mu \mathrm{CT}, \mathrm{x} \text {-ray, Osteoclast } \\
\text { quantification \& } \\
\text { histomorphometrical } \\
\text { analysis }\end{array}$ & $\begin{array}{l}\text { Mandibular } \\
\text { bone \& tooth }\end{array}$ & $\begin{array}{l}\text { SF might even affect non- } \\
\text { weight-bearing bones }\end{array}$ \\
\hline $\begin{array}{l}\text { (Gerbaix et al. } \\
\text { 2018) }\end{array}$ & $\begin{array}{l}\text { Bion-M1 } \\
30 \text { days }\end{array}$ & $\begin{array}{l}\mathrm{N}=12 \\
\text { Mice, } \\
\mathrm{M}\end{array}$ & $\begin{array}{l}\text { SF: } n=5, \text { SF-R: } \\
n=5 \\
\text { Habitat control: } \\
n=6 \text {, Control: } \\
n=12\end{array}$ & $\mu \mathrm{CT}$ & $\begin{array}{l}\text { CAL, } \\
\text { navicular, talus }\end{array}$ & $\begin{array}{l}\text { Reduction in cortical } \\
\text { thickness at the point of } \\
\text { ground contact bone sites } \\
\text { muscle insertions }\end{array}$ \\
\hline
\end{tabular}


Table 6. (continued)

\begin{tabular}{|c|c|c|c|c|c|c|}
\hline $\begin{array}{l}\text { (Maupin et al. } \\
\text { 2019) }\end{array}$ & $\begin{array}{l}\text { ISS, } \\
\sim 28 \text { days }\end{array}$ & $\begin{array}{l}\mathrm{N}=30 \\
\text { Mice } \\
\mathrm{M}\end{array}$ & $\begin{array}{l}\mathrm{SF}: \mathrm{n}=10, \mathrm{GC}: \\
\mathrm{n}=10 \text {, baseline: } \\
\mathrm{n}=10\end{array}$ & Skeletal maturation & $\begin{array}{l}\text { FEM, tibia, \& } \\
\text { vertebrae }\end{array}$ & \\
\hline $\begin{array}{l}\text { (Fitzgerald et } \\
\text { al. 2019) }\end{array}$ & $\begin{array}{l}\text { Bion-M1 } \\
30 \text { days }\end{array}$ & $\begin{array}{l}\mathrm{N}=28 \\
\text { Mice, } \\
\mathrm{M}\end{array}$ & $\begin{array}{l}\text { SF }: n=5 \\
\text { SF-VIV: } n=8 \\
\text { GC: } n=7 \\
\text { GC-VIV: } n=7\end{array}$ & Histological analysis & $\mathrm{AC}, \& \mathrm{SC}$ & $\begin{array}{l}\text { Weight-bearing AC was } \\
\text { negatively affected }\end{array}$ \\
\hline
\end{tabular}

Table 7. Exercise Countermeasures - Study characteristics

\begin{tabular}{|c|c|c|c|c|}
\hline Author, year & $\begin{array}{l}\text { Mission \& } \\
\text { subjects }\end{array}$ & Countermeasure Device & $\begin{array}{c}\text { Exercise regimen } \\
\text { (mode, intensity, volume, } \\
\text { duration) }\end{array}$ & Main Outcomes \\
\hline (Thornton 1977) & $\begin{array}{l}\text { Skylab } 2: 28 \text { days, } \\
n=3 . \text { Skylab } 3: 56 \\
\text { days, } n=3 . \text { Skylab } \\
4: 84 \text { days, } n=2\end{array}$ & $\begin{array}{l}\text { Skylab 2: Cybex } \\
\text { Isokinetic Dynamometer, } \\
\text { bicycle ergometer. } \\
\text { Skylab 3: MK-I \& MK- } \\
\text { II. Skylab 4: A Treadmill } \\
\text { was added. }\end{array}$ & $\begin{array}{l}\text { The crew members used } \\
\text { their favorite maneuvers. } \\
\text { On Skylab 3, the use of } \\
\text { cycle ergometer doubled. }\end{array}$ & $\begin{array}{l}\text { Exercise reduced the loss of } \\
\text { muscle volume and strength, as } \\
\text { well as body mass }\end{array}$ \\
\hline $\begin{array}{l}\text { (Goodship et al. } \\
\text { 1998) }\end{array}$ & $\begin{array}{l}\text { EuroMir-95 } \\
\mathrm{N}=1\end{array}$ & Impulsive loading device & $\begin{array}{l}500 \text { cycles per day using a } \\
\text { mechanical loading device. }\end{array}$ & $\begin{array}{l}\text { A mechanical loading device } \\
\text { can mitigate the loss of BMD }\end{array}$ \\
\hline (LeBlanc et al. 2000) & $\begin{array}{l}\text { Mir, } 12 \text { missions } \\
(1990-1995) \\
126-197 \text { days }\end{array}$ & $\begin{array}{l}\text { Bungee cord, bicycle } \\
\text { ergometer, treadmill }\end{array}$ & $\begin{array}{l}3 \mathrm{~d} / \text { wk mandatory exercise. } \\
2 \text { sessions of } 1-1.5-\mathrm{hr} / \mathrm{d} . \\
\text { Cycle ergometer: HR of } \\
160-180 \text { (bpm). } \\
\text { Strength training: } \\
10-30 \mathrm{~min} / \mathrm{d} .\end{array}$ & $\begin{array}{l}\text { Significant reduction of bone } \\
\text { mineral was observed in the } \\
\text { spine despite exercise } \\
\text { countermeasure }\end{array}$ \\
\hline $\begin{array}{l}\text { (Guilliams et al. } \\
\text { 2003) }\end{array}$ & $\begin{array}{l}\text { ISS, 129-196 } \\
\text { days } \\
\mathrm{N}=6\end{array}$ & Unstated & Unstated & $\begin{array}{l}\text { No significant differences in } \\
\text { muscle strength observed, but } \\
\text { decreases in body weight were } \\
\text { observed }\end{array}$ \\
\hline (Trappe et al. 2009) & $\begin{array}{l}\text { ISS, } 180 \text { days } \\
N=9\end{array}$ & TVIS, CEVIS, iRED & $\begin{array}{l}6 \mathrm{~d} / \mathrm{wk} .7 \% \text { to } 10 \% \text { of } \\
\text { waking time spent } \\
\text { exercising. } \\
\text { Cycle ergometer: } 138 \pm 26 \\
\text { min/w. Treadmill: } 146 \pm 34 \\
\text { min/w. } \\
\text { Resistance: } 3-6 \mathrm{~d} / \mathrm{wk}, 3-6 \\
\text { sets of } 12-20 \mathrm{reps} \text { for each } \\
\text { leg exercise. }\end{array}$ & $\begin{array}{l}\text { Muscle mass loss was slightly } \\
\text { hindered by exercise } \\
\text { countermeasures }\end{array}$ \\
\hline $\begin{array}{l}\text { (Cavanagh et al. } \\
\text { 2010) }\end{array}$ & $\begin{array}{l}4 \text { missions to the } \\
\text { ISS, } 181 \pm 15 \\
\text { days } \\
\mathrm{N}=4\end{array}$ & TVIS, CEVIS, iRED & $\begin{array}{l}\text { Two devices per session: } \\
\text { TVIS: } 23.86 \pm 7.22 \mathrm{~min} . \\
\text { CEVIS: } 28.5 \mathrm{~min} . \\
\text { iRED: } 19 \pm 10.7 . \\
\text { Total time under load: } \\
43.11 \pm 15 \text { min. }\end{array}$ & $\begin{array}{l}\text { Exercise duration and/or } \\
\text { loading applied to the LE } \\
\text { could not prevent reductions in } \\
\text { BMD }\end{array}$ \\
\hline (Fitts et al. 2010) & $\begin{array}{l}\text { ISS increments } 5 \\
\text { to } 11,177 \pm 4 \\
\text { days } \\
\mathrm{N}=9\end{array}$ & $\begin{array}{l}\text { TVIS, CEVIS, Velo } \\
\text { (Russian cycle } \\
\text { ergometer), iRED }\end{array}$ & $\begin{array}{l}\text { TVIS: } 146 \pm 32 \mathrm{~min} / \mathrm{w} . \\
\text { CEVIS: } 138 \pm 26 \mathrm{~min} / \mathrm{w} . \\
\text { iRED: } 3-6 \mathrm{~d} / \mathrm{wk} \text { of squats, } \\
\text { heel raises, } \& \text { deadlifts at } 12 \\
\text { sets of } 20 \text { reps. }\end{array}$ & $\begin{array}{l}\text { Exercise countermeasures } \\
\text { were incapable of providing } \\
\text { the intensity and wide range of } \\
\text { motion needed to prevent } \\
\text { muscle atrophy }\end{array}$ \\
\hline
\end{tabular}


Table 7. (continued)

\begin{tabular}{|c|c|c|c|c|}
\hline $\begin{array}{l}\text { (Gopalakrishnan } \\
\text { et al. 2010) }\end{array}$ & $\begin{array}{l}\text { ISS, } 181 \pm 15 \\
\text { days } \\
\mathrm{N}=4\end{array}$ & TVIS, CEVIS, iRED. & $\begin{array}{l}\text { 2-7 d/wk: Deadlift, leg heel } \\
\text { raises, squats on the iRED. } \\
\text { TVIS: } 1-5 \mathrm{~d} / \mathrm{wk}, 15-35 \\
\mathrm{~min} / \mathrm{d} \text {. CEVIS: } 2-6 \mathrm{~d} / \mathrm{wk} \text {, } \\
\text { 24-41 } \mathrm{min} / \mathrm{d} \text {. }\end{array}$ & $\begin{array}{l}\text { Exercise countermeasures } \\
\text { were insufficient to replicate } \\
\text { the daily mechanical loading } \\
\text { experienced on Earth }\end{array}$ \\
\hline (Smith et al. 2012) & $\begin{array}{l}\text { ISS expeditions } \\
\text { 14-20, } \mathrm{N}=13 \\
\text { iRED: }(6 \mathrm{M}, 2 \mathrm{~F}) \\
\text { ARED: }(3 \mathrm{M}, 2 \mathrm{~F})\end{array}$ & $\begin{array}{l}\text { TVIS, CEVIS, iRED, } \\
\text { ARED }\end{array}$ & $\begin{array}{l}\text { Similar exercise protocol } \\
\text { for both groups: } 6 \text { days per } \\
\text { week of treadmill or cycle } \\
\text { for } 30 \text { minutes, plus } \\
\text { resistive exercise (squats, } \\
\text { deadlifts, heel raises) using } \\
\text { iRED or ARED. }\end{array}$ & $\begin{array}{l}\text { Improvements in nutrition and } \\
\text { resistance exercise regimens } \\
\text { combined could reduce BMD } \\
\text { deficits }\end{array}$ \\
\hline (Fitts et al. 2013) & $\begin{array}{l}\text { ISS missions } \\
(2002-2005) \\
N=9\end{array}$ & CEVIS, TVIS, iRED & $\begin{array}{l}\text { CEVIS: } 138 \pm 26 \mathrm{~min} / \mathrm{w} \text { at } \\
126 \pm 10 \mathrm{~W} . \text { TVIS: } 146 \pm \\
32 \mathrm{~min} / \mathrm{w} \text { at } 3.2 \pm 0.5 \mathrm{mph} \text {. } \\
\text { iRED: } 12 \text { sets of } 20 \mathrm{reps}, 3- \\
6 \mathrm{~d} / \mathrm{wk} \text {, of squats, heel } \\
\text { raises, \& deadlifts. }\end{array}$ & $\begin{array}{l}\text { Enzyme capacity could be } \\
\text { maintained by an adequate } \\
\text { treadmill exercise } \\
\text { countermeasure }\end{array}$ \\
\hline (English et al. 2015) & $\begin{array}{l}\text { ISS } 1-25 \\
\mathrm{~N}=37\end{array}$ & $\begin{array}{l}\text { iRED, ARED, CEVIS, } \\
\text { TVIS }\end{array}$ & Unstated & $\begin{array}{l}\text { The ARED might be better } \\
\text { suited to prevent losses in } \\
\text { strength compared to the iRED }\end{array}$ \\
\hline $\begin{array}{l}\text { (Laughlin et al. } \\
\text { 2015) }\end{array}$ & $\begin{array}{l}\text { ISS } 1-25 \\
\mathrm{~N}=32 \\
26 \mathrm{M}, 6 \mathrm{~F}\end{array}$ & $\begin{array}{l}\text { CEVIS, TVIS \& T2, } \\
\text { Velo, iRED, ARED }\end{array}$ & $\begin{array}{l}2.5 \mathrm{hr} / \mathrm{d}, 6 \mathrm{~d} / \mathrm{wk} .1 \mathrm{hr} \text { of } \\
\text { aerobic \& } 1.5 \mathrm{hr} \text { of } \\
\text { resistance training. }\end{array}$ & $\begin{array}{l}\text { The exercise countermeasure } \\
\text { was somewhat effective in } \\
\text { maintaining muscle strength \& } \\
\text { endurance }\end{array}$ \\
\hline (Smith et al. 2015) & $\begin{array}{l}\text { ISS 14-35 } \\
\text { iRED: } \sim 173 \text { days, } \\
\text { ARED: } \sim 148 \\
\text { days, } \\
\mathrm{N}=23\end{array}$ & $\begin{array}{l}\text { iRED, ARED, TVIS, } \\
\text { CEVIS }\end{array}$ & Unstated & $\begin{array}{l}\text { Bone loss can be mitigated } \\
\text { through a combination of } \\
\text { exercise-induced increased } \\
\text { bone formation \& } \\
\text { bisphosphonates }\end{array}$ \\
\hline (Hides et al. 2016) & $\begin{array}{l}\text { ISS, } 180 \text { days, } \\
\mathrm{N}=1 \mathrm{M}\end{array}$ & $\begin{array}{l}\text { ARED, T2 or BD- } 2, \& \\
\text { CEVIS or VELO }\end{array}$ & Unstated & $\begin{array}{l}\text { Exercise countermeasures } \\
\text { were somewhat effective in } \\
\text { preventing trunk muscle mass } \\
\text { reduction \& restoring balance }\end{array}$ \\
\hline
\end{tabular}

\section{Discussion}

\section{Skeletal muscle responses to microgravity in humans}

Effects of spaceflight on human skeletal muscle

have been found to vary substantially by the sites examined, generally trending toward increased effects in the inferior direction, e.g., the lower spine and legs. For example, McNamara et al. (2019) explored the effects of spaceflight on the neck musculature of astronauts in longduration missions of four to six months. Outcomes indicated that the muscle CSA and fatty infiltration of crew members using the Advanced Resistive Exercise Device (ARED) changed following $\mu \mathrm{G}$. Significant muscle CSA increases were noted in the rhomboid minor (23.1\%) at T1-T2, sternocleidomastoid (9.0\%) at C4-C5, in the trapezius $(25.1 \%)$ at $\mathrm{C} 6-\mathrm{C} 7$, and in the semispinalis capitis $(11.5 \%)$ at $\mathrm{C} 4-\mathrm{C} 5$. This is presumably due to exercise countermeasures. There has been evidence of strength reduction in the arms, however, as shown in a study by Puglia et al. (2018), in which two subjects who participated in separate missions (180 and 191 days) were examined. The results showed that in general, isometric handgrip and precision pinch force decreased during long-term spaceflights. Thus, findings indicate that long-duration spaceflight could be detrimental to astronaut health and efficacy for tasks requiring full arm strength and functionality.

Crucial to general functional performance, of course, is the integrity of the spinal musculature. Oganov et al. (1991) measured spinal muscle volume changes in cosmonauts before and after prolonged flights. The researchers showed that, on average, the volume and density of back muscles slightly decreased. The authors suggested that the relative stability of muscle volume may have been due to the effectiveness of countermeasures. These data showed, however, that 
exercise during flight did not completely prevent negative changes in spinal muscles. Considering changes in both spinal muscles and intervertebral discs, Chang et al. (2016) examined astronauts who participated in prolonged space missions. Results showed a decrease in lumbar paraspinal Functional Cross-Sectional Area (FCSA) by $19 \%$ during post-flight assessments. Lumbar lean muscle FCSA percentage decreased from $86 \% \pm 5 \%$ to $72 \pm 7 \%$ when tested immediately post-flight. Researchers mentioned that, although subjects participated in standard pre-flight, inflight, and post-flight exercise programs, results showed lumbar paraspinal muscle atrophy, likely due to the lack of exercise countermeasures that target the lumbar paraspinal muscles.

Bailey et al. (2018) sought to determine changes in a variety of lumbar spine aspects in subjects on board the International Space Station (ISS) for approximately six months. Results showed that the average decrease in lumbar lordosis was about $11 \%$. Total lumbar vertebral wedging decreased by $13 \%$ and $23 \%$, respectively, in two subjects. Active flexion-extension range of motion decreased by $22.1 \%, 17.3 \%$, and $30.3 \%$ for L2-L3, L3-L4, and L4-L5, respectively. Regarding muscle atrophy, five subjects showed a $20 \%$ average decrease in FCSA and $8 \%$ to $9 \%$ average decrease in CSA for both multifidus (MF) and erector spinae (ES). Overall, MF atrophy was found to be responsible for lumbar flattening and increased stiffness.

Burkhart et al. (2019) expanded the spinal inquiry to include lumbopelvic musculature. Based on the results of this study, following four- to seven-month spaceflight, CSA of the ES, MF, and quadratus lumborum $(\mathrm{QL})$ decreased $4.6 \%$ to $8.4 \%$, immediately post-flight, but fully recovered after one year. Paraspinal muscle attenuation (density) at the same time point was negatively affected by $-5.9 \%$ to $-8.8 \%$ and continued to be significantly lower than preflight values after two to four years. McNamara et al. (2019) also studied changes in lumbopelvic muscles (PS, QL, ES, and MF) due to prolonged spaceflight. Results revealed that 14 of the 16 astronauts experienced a reduced volume of all their lumbopelvic muscles, ranging from $2.4 \%$ to $10.5 \%$, after exposure. On average, the lumbopelvic muscles of crew members significantly decreased by $5.1 \%$ following $\mu \mathrm{G}$. Individual muscle volume and attenuation changes were also noted in the ES and MF by $5.3 \%$, and in the QL by $9.5 \%$ following spaceflight. Significant PS muscle volume and attenuation changes on the other hand, were not observed.
Lumbopelvic muscles are involved in a significant amount of weight-bearing, but the lower limbs may be equally loaded, if not more, during daily terrestrial activities. The legs can also be isolated and examined functionally in ways that pelvic and spinal muscles cannot. In a study by Antonutto et al. (1995), the effects of 31 days of $\mu \mathrm{G}$ on the maximal explosive power (MEP) of the lower limb muscles in one astronaut was tested. When tested after landing, the mean force, maximal velocity, maximal power (Wpeak and Wmean), maximal acceleration, and overall mechanical work decreased between $60 \%$ and $80 \%$ of pre-flight values. Antonutto et al. (1998) confirmed the previous findings that functional capacity of lower limbs is negatively affected by spaceflight, again in one astronaut. They demonstrated that the MEP declines after spaceflight corresponds to a similar decline in EMG activity of the Quad muscles. In comparison to the previous study, after 21 days of spaceflight, a larger decline in the Wpeak and Wmean was observed. The researchers suggested that individual characteristics and/or in-flight exercise training were responsible for these differences. Furthermore, Antonutto et al. (1999) later studied four astronauts (S1, S2, S3, S4) before and after spaceflight missions ranging from 31 to 180 days. When tested two days after landing, MEP dropped to about $68 \%$ of preflight value for S1, who spent 31 days in space. MEP fell to about $50 \%$ of preflight value for S2, S3, and S4, who spent between 169 to 180 days in space. Maximal force value (Fpeak) decreased by $11.7 \%, 26.2 \%, 31.5 \%$, and $27.0 \%$ for $\mathrm{S} 1$, S2, S3, and S4, respectively. Peak velocity (Vpeak) was reduced by $24.2 \%$ for S1, while Vpeak fell by $27.8 \%$ to $35.8 \%$ for S2, S3, and S4. Lastly, muscle mass of the lower limbs for all subjects decreased by $9 \%$ to $13 \%$. It was evident that the decrease in muscle mass was much smaller than the decrease in the MEP in the investigated spaceflight durations. The authors suggested that complex mechanisms must be responsible for the observed decline in power output.

Some variability in spaceflight effects on lower limb muscles has been found, given their functional roles. Tesch et al. (2005) investigated the effects of spaceflight on the knee extensor and flexor muscle size and function of astronauts. Pre-and post-flight data indicated that astronauts showed a significant decrease in maximal voluntary contraction (MVC) of $10 \%$. Concentric force was reduced by $9 \%$, while eccentric force was reduced by $11 \%$. CSA of the gluteal and quadriceps (Quad) muscles was significantly reduced by $8 \%$, but no 
reduction was found in knee flexors, which are less involved in anti-gravity functions. The researchers estimated that the knee extensors decreased in strength at a rate of 3-4 \% per week during spaceflight. Despite the previous findings of insignificant changes to knee flexors, Akima et al. (2000) complicated the conversation. Three astronauts (A, B, C) flew nine, 15, and 16 days in space. The results showed that subjects $\mathrm{A}$ and $\mathrm{C}$ experienced the most significant decrease in knee extensor volume postflight $(15.4 \%$ and $11.6 \%$, respectively). For knee flexors, the greatest decrease in volume occurred four days after landing for subjects $\mathrm{A}$ and $\mathrm{C}(14.1 \%$ and $8.6 \%$, respectively) and one day after landing for subject B $(11.6 \%)$. The greatest changes in Plantaris (PL) volume were approximately $-12 \%$ for subject $\mathrm{A}$, approximately $-8 \%$ for subject $\mathrm{B}, \quad$ and approximately $-15 \%$ for subject $\mathrm{C}$. Overall, muscle atrophy (normalized by flight duration) ranged from $0.62 \%$ to $1.04 \%$ per day. These findings suggest that muscle atrophy varies among individuals and muscle groups. Edgerton et al. (1995) conducted a microscopic study of knee extensors, examining fiber size and enzymatic properties of the VL muscle, measured by biopsy of eight astronauts, three who underwent two fiveday flights, and five who underwent one flight for 11 days. For the astronauts involved in the 11-day mission, the quantity of type I fibers decreased by approximately $6 \%$ to $8 \%$, and the CSA decreased by $16 \%$ in one flight. CSA decreased by $23 \%$ and $36 \%$ in fiber types IIA and IIB, respectively. The mean number of capillaries per fiber for fiber type I decreased by $19 \%$, while for fiber type IIA, CSA decreased by $25 \%$, and for fiber type IIB it decreased $26 \%$. For type I fibers, the average ratio of $\alpha$-glycerophosphate dehydrogenase (GPD) to SDH activity among all eight participants increased by $85 \%$. ATPase increased by $9 \%$ and the ratio of ATPase to $\mathrm{SDH}$ increased by $38 \%$ for type II fibers. Findings suggest that short-duration flights can affect muscle fibers, including their thickness, metabolism, and blood supply.

The lower leg is an equally complex story. Trappe et al. (2001) showed that changes in maximal isometric calf muscle strength, muscle force-velocity properties, fiber composition, or size of the calf muscles were not significant for either bed rest or spaceflight after 17 days. Despite the non-significant outcomes, it is possible that the extensive in-flight testing of ankle plantar flexor function worked as a countermeasure and preserved the muscle. Despite the findings in Trappe et al. study, significant changes in lower leg muscles were found by other authors. Riley et al. (2000) examined the density and length of thin filaments of the SOL muscle fibers of astronauts. Outcomes indicated that following 17 days of spaceflight, CSA of muscle fibers in the SOL were reduced by $15 \%$. Myofibrils post-flight were slimmer, as the $\mathrm{Z}$ bands were shorter in length. Sarcomeres remained the same, as they did not lose any myofilaments. The density of the myofilament varied with sarcomere length. The thickness of filament density and filament spacing remained unchanged from spaceflight. Compared with the pre-flight controls, the thin filaments following space travel decreased significantly by $17 \%$ in density when close to the $\mathrm{Z}$ band. Similarly, the mid-I band value was reduced by $21 \%$, and the A band density dropped significantly by $26 \%$. Fibers after spaceflight had a $22 \%$ decrease in thickness at the overlap A band region, compared to the $\mathrm{Z}$ band area. This combination suggests that atrophied muscle fibers risk damage to sarcomeres after landing.

For the first time, Fitts et al. (2010) presented the cellular analysis of the effects of long-duration spaceflight (180 days) on the structure and function of human skeletal muscle. Findings revealed that relative to the pre-flight values, fiber mass, force, and power decreased in SOL type I, SOL type II, gastrocnemius (GAS) type I, and GAS type II, respectively. Substantial loss of fiber CSA was evident in the SOL type I by $20 \%$. Similarly, the most considerable decrease in peak force occurred in SOL type I by $35 \%$. Crew members with the highest percentage decline in mean fiber diameter showed the greatest increase in the number of fast types II fibers. A significant difference between individuals was observed in both the degree of fiber atrophy and the loss of peak force.

Gopalakrishnan et al. (2010) examined the lower and upper extremity of four male astronauts before and after long-duration spaceflight. Regarding the volume and strength of skeletal muscles, the greatest decreases in muscle volume were in the SOL $(19 \pm 7 \%)$, GAS $(10 \pm 5 \%)$, and ankle dorsiflexor muscles $(10 \pm 3 \%)$. Some losses were also found in the thigh muscles (knee extensors: $6 \pm 3 \%$, knee flexors: $7 \pm 4 \%$, adductors: $4 \pm 3 \%$ ). Slight or no volume decreases were detected in the arm muscles. Isokinetic strength decreased in knee flexors $(24 \pm 8 \%)$ and extensors $(10 \pm 11 \%)$. Ankle PL strength decreased by $22 \pm 6 \%$, while dorsiflexor strength decreased $8 \pm 16 \%$. Isometric strength was reduced $20 \pm 16 \%$ in PL and $4 \pm 22 \%$ in dorsiflexors. Knee 
extensor strength reduced $15 \pm 13 \%$, and flexor strength reduced $20 \pm 17 \%$. Hip extensor isometric strength reduced $15 \pm 26 \%$, and hip flexors reduced $28 \pm 9 \%$.

Adding a functional layer to the calf muscle findings, Lambert et al. (2001) examined contractile and elastic features of the PL muscles of 14 cosmonauts in space for 90 to 180 days. Outcomes revealed that spaceflight significantly reduced the maximal isometric torque by $17 \%$ in all participants. Maximal shortening velocity (Vmax), as well, showed a significant increase by $31 \%$ between pre-and post-flight. Maximal muscle activation decreased by $39 \%$. Musculotendinous stiffness showed a $25 \%$ increase. During passive conditions, whole joint stiffness reduced significantly by $21 \%$, but it was retained under active conditions following spaceflight. All subjects had access to a cycle ergometer, a treadmill, and devices for resistance training, but no exercise protocol was recorded.

Koryak (2019) elaborated on calf muscle functioning in eight cosmonauts in flights of $213.0 \pm$ 30.5 days. Architectural and contractile properties of the triceps surae (TS) muscle were measured pre- and postflight. Variables included MVC, tetanic tension (Po), voluntary and electrically evoked contraction times and force deficiency (Pd), fiber length (Lf), and Muscle thickness (Hm). A four-day cycle was used for the exercise regimen, which is later discussed in the Effectiveness of exercise countermeasure on the musculoskeletal system section. Results found the maximum voluntary isometric contraction (MVIC) decreasing $41.7 \%$, Po decreasing by $25.6 \%$, and $\mathrm{Pd}$ increasing by $49.7 \%$. The time from the moment of stimulation to peak twitch (TPT) saw a $4 \%$ increase. The time from contraction peak to half-relaxation (1/2 RT) decreased by $17.6 \%$. Electromechanical delay (EMD) increased by $34 \%$. Lf decreased by $22.5 \%$ in gastrocnemius medialis (GM), $35.2 \%$ in gastrocnemius lateralis (GL), and $28.1 \%$ in the SOL. Hm decreased by $18.9 \%$ in the GM, $19.8 \%$ in the LG, and $18.8 \%$ in the SOL. The researcher suggested that considering the specific functions of individual calf muscles is important in planning effective countermeasures to microgravityinduced changes.

\section{Skeletal muscle responses to microgravity in animals}

Many studies have examined the effects of spaceflight on the measurable external phenomena of animal skeletal muscles, from the cellular level to overall mass. Spaceflight-induced adaptations in skeletal muscle vary substantially by the sites examined, generally showing severe effects in the antigravity muscles such as the VI and SOL. Baldwin et al. (1993) showed that after nine days aboard Spacelab Life Science 1 (SLS-1), flight rats significantly reduced VI and VL muscle weight relative to that of control rats. However, the most significant difference $(23 \%)$ in muscle weight was found in the VI, which is known as an antigravity muscle with a high proportion of slow fibers. Palmitate oxidation capacity had a significant reduction of $37 \%$ in the low and high oxidative areas of the vastus muscle. In the same flight, Haddad et al. (1993) revealed that the VI was atrophied significantly $(\sim 22 \%)$ in the flight group as opposed to the tibialis anterior (TA) and VL. This atrophy was associated with a net decrease in type I MHC protein by $40 \%$ and in type IIa by $19 \%$ in the VI muscle. Conversely, a net increase of type IIb and IIx MHC expression was found following spaceflight exposure. This was one of the first reports demonstrating that mRNA expression levels showed a similar pattern as that observed in MHC protein expression of the VI and the red region VL muscles. Furthermore, Kraemer et al. (2000) examined the effects of 10 days of spaceflight on rats' fast-twitch muscle fibers (types I, IC, IIC, IIA IIAD, IID, IIDB, IIB) of the GAS and Extensor Digitorum Longus (EDL) muscles. Measures of the CSA and MHC proportions revealed that following spaceflight, muscle mass was significantly reduced by $\sim 11 \%$ in spaceflight animals for the GAS and $5 \%$ for the EDL muscles. Muscle atrophy was also noted in individual fibers of the EDL (fiber type IIB) and of the GAS (fiber types I, IIA, IIDB, and IIB) at a significant level. In the EDL, there was a significant reduction in MHC IId content. Similarly, the GAS muscle showed significantly reduced MHC IId content in the superficial or white region; however, it did not show any changes in the deep or red regions. In summary, the EDL and GAS muscles exposed to short-term $\mu \mathrm{G}$ demonstrated rapid plasticity.

Substantial research has been conducted focusing on the hindlimbs of rats or mice. During the SLS-2 shuttle mission, Hansen et al. (2004) compared the effects of 14-day spaceflight observed in rat hindlimb muscles with the effects of spaceflight on the rat diaphragm (DIA). The results revealed that the negative structural and metabolic changes noted in rats' hindlimb muscles do not occur in the DIA. Two hindlimb muscles, the GM and TA, on the other hand, presented slow-to-fast transitions of fiber types. Most notably, IIDB fibers of the GM were reduced by $59 \%$, with an increase of $51 \%$ in 
IIB fibers. Similarly, IIDB and IIB fibers of the TA increased, while the relatively slower IIA fibers decreased by $33 \%$. Similarly, Schuenke et al. (2009) also showed that while 14 days of gravitational unloading was associated with decreases of $8 \%$ to $9 \%$ in muscle mass of the EDL and GAS, no significant changes were noted in the DIA. $\mu \mathrm{G}$ was not found to have a substantial effect on the relative makeup of fiber types, except for a $9.5 \%$ decrease in IID fibers of the EDL. The fast-twitch fibers in the hindlimbs were not found to undergo slow-to-fast or fast-to-faster transformations of fiber types to any significant degree. The CSA of the EDL and GAS displayed significant atrophy of $10 \%$ to $35 \%$, with deep red GAS atrophying the most. Unlike other hindlimb fibers studied, fibers of type IID and IIDB in the EDL did not display significant atrophy in the CSA.

The antigravity and postural muscle, the soleus (SOL), is known for its primary slow phenotype and susceptibility to unloading and disuse. Therefore, it has been used often in unloading studies to investigate skeletal muscle atrophy in $\mu \mathrm{G}$. One particularly early SOL study is that of Gurovsky et al. (1980). Using the Soviet biosatellite Cosmos-936, the researchers reported significant atrophy in the leg muscles, particularly SOL muscle, and metabolic changes among animals exposed to 18.5-day weightlessness. These findings were strengthened ten years later in the study of Musacchia et al. (1990), examining the effect of a seven-day spaceflight on SOL and EDL muscles of adult male rats. Compared with their vivarium controls, the researchers showed that the absolute muscle mass in the spaceflight animals was reduced by $20 \%$ in the SOL and $\sim 15 \%$ in the EDL muscles. The CSA of slow- and fast-twitch fibers showed a significantly reduced size and an increased fiber density and capillary density in the SOL and EDL muscles compared to the control groups. It was shown that the antigravity SOL was more affected than the EDL in terms of weightlessness unloading.

In Miu et al. (1990), the metabolic properties and size of muscle fibers after 12.5 days aboard Cosmos 1887 were measured on the deep and superficial areas of the SOL and GM in rats. In summary, the histochemical assays showed that muscle atrophy in SOL fibers was twofold more than in the GM fibers. The fibers located in the deep (having the highest percentage of slow-twitch fibers) region of the GM atrophied more than the fibers located in the superficial region (having the lowest percentage of slow twitch fibers) of the muscle. During Cosmos 1514 and Cosmos 1667, Holy et al. (1991) examined the effects of five-day and seven-day spaceflights on the contractile protein properties of skinned muscle fibers of rats' SOL, PL, and GL muscles. These muscles were chosen because the SOL has $87 \%$ slow-twitch fibers, while the PL and the GL are fasttwitch muscles containing, respectively, $9 \%$ and $7 \%$ slow oxidative fibers. This study demonstrated that spaceflight could affect the mechanical properties, precisely, muscle tension and diameter, of muscle fibers, particularly in the case of slow-twitch fibers. The diameter of the SOL muscle fibers decreased by $14 \%$ after the seven-day spaceflight. There was a small significant decrease in GAS fiber size after the five-day spaceflight. The PL muscles exhibited no significant change in fiber diameter after both flights. Maximal tensions decreased in the SOL muscle up to $28 \%$ after the seven-day spaceflight, while they did not change in the GAS and PL muscles. Due to the nature of the changes in muscle tension and the observed decline in muscle mass/diameter of spaceflight groups, the results of this study point likewise towards muscle atrophy.

Examining the enzymatic activities and morphological response of the SOL muscle fibers, Ohira et al. (1992) observed changes in rats aboard the 14-day Cosmos 2044, similar to those from Cosmos 1887. The flight rats experienced a $25 \%$ reduction in muscle mass relative to the control, while the CSA was reduced more significantly in slow-twitch $(30 \%)$ than in fast-twitch fibers. Also, the CSA of fast fibers and fibers with both a slow and fast MHC of the experimental group was $16 \%$ to $37 \%$ lower than the control group. The enzymatic activities of SDH and GPD were relatively unchanged. The total SDH activity (SHD*CSA), showed a significant reduction in the slow fibers, mainly due to atrophy in the muscle fibers. In another study during Cosmos 1887, Bell et al. (1992) showed the selective response of mitochondrial enzymes to spaceflight. The authors investigated the effects of a 12.5-day spaceflight on the $\mathrm{SDH}$ distribution of fast-twitch oxidative-glycolytic (FOG) and slow-twitch oxidative (SO) fibers in rat SOL muscle, relative to ground-based simulated flight condition. Results indicated that the mean SDH distribution in all flight and simulated flight control muscle fibers was significantly greater in the subsarcolemmal region than in the intermyofibrillar region. These findings suggest that when determining the influence of spaceflight on muscle fiber oxidative enzymes, it is vital to consider the location of the enzyme within a fiber. 
Caiozzo et al. (1994) investigated the correlation between changes in the SOL muscle phenotype and its functional properties. The SOL muscles of six mice flown on board the Shuttle Transport System-54 (STS-54) for six days were compared with a ground-based control group. The SOL of the flight animals was found to weigh $27 \%$ less than the controls; the flight group's overall body weight was $7 \%$ less than that of the control group. There was a reduction in slow type I fiber CSA by approximately $24 \%$, and in type II by $3 \%$ in the flight group. It was observed that the flight SOL muscles expressed the fast type IIx MHC isoform, while this was not evident in any of the control muscles. In the flight group, the mean value of the absolute maximal isometric tension was found to be $28 \%$ lower than that of the control group. These findings showed that the SOL muscle became weaker and faster in terms of structure and function.

In Riley et al. (1996), in-flight and post-flight alterations of the SOL, adductor longus (AL), and EDL muscles were examined in rats in the SLS-1 (nine days) and SLS-2 (14 days). Reduction of muscle-weight-tobody-weight ratios in the SLS-1 flight was measured at $30 \%, 29 \%$, and $12 \%$ for the SOL, AL and EDL muscles, respectively; in the SLS-2 flight, the ratios for the SOL, AL and EDL muscles were observed to be reduced by $38 \%, 47 \%$, and $24 \%$, respectively. Additionally, the $\mathrm{AL}$ and SOL muscles showed a decrease of myofiber areas by $42 \%$ and $26 \%$ after 14 days of spaceflight. The percentage myofiber atrophy was higher in the $\mathrm{AL}$ than those in the SOL because myofibers were measured in the AL muscle's slow region, where it is more susceptible to atrophy. For the nine-day flight rats, the $\mathrm{AL}$ and $\mathrm{SOL}$ myofiber areas decreased by $31 \%$ and $32 \%$, respectively, while EDL was not atrophied. Simultaneously, Allen et al. (1996) indicated a significant reduction in the SOL myonuclear number and size of fibers expressing type I MHC. These findings suggest that changes in the number of myonuclei may contribute to the reduction in fiber size associated with chronic unloading of the musculature.

Caiozzo et al. (1996) investigated the influence of a 14-day spaceflight (STS-58) on the contractile properties, MHC protein, and mRNA protein isoforms of rats' SOL, VI, TA, and PL muscles. Results indicated that, compared with controls, the twitch tension and TPT of SOL in the flight subjects were reduced significantly by $33 \%$ and $20 \%$, respectively. Moreover, the forcevelocity relationship in the SOL varied considerably. It was higher in Vmax by $20 \%$ but lower in maximal isometric tension by approximately $36 \%$. Consequently, SOL activity was adequate in the low-force region due to increased Vmax but at a disadvantage in the high-force area because of the substantial decrease in strength. Further, in terms of MHC protein content, in SOL muscle, slow type I was significantly lower (30\%) than in the control group, while the fast type IIX was significantly higher. In flight VI muscles, fast type IIA fibers were significantly reduced; however, the fast type IIX was significantly increased. In the PL and TA muscles, MHC isoforms remained constant. Regarding the mRNA isoform content, the fast type IIX in the flight group of the SOL was significantly higher than the control group by $970 \%$. Similarly, the mRNA content in the fast type IIX and IIB of the VI muscles of the flight group were also higher than controls (225\% and $288 \%$, respectively). However, reductions of mRNA content in VI muscle in the flight group were noted in slow type I by $32 \%$ and fast type IIA by $39 \%$. The fast type IIB in the PL and TA muscles of the flight group also exhibited elevated mRNA isoform content but no changes at the protein level. Findings revealed that spaceflight relatively alters muscle mass, as well as the contractile properties, MHC, and mRNA isoform contents of rat muscles.

In another prominent study, Martin et al. (1988) evaluated the effects of seven days $\mu \mathrm{G}$ on rat SOL, EDL, $\mathrm{AL}, \mathrm{GM}$, and PL muscles. The results revealed that all of the flight muscle weights decreased relative to their controls, with the SOL exhibiting the highest weight reduction and the EDL showing the lowest reduction. The highest decrease in fiber CSA was observed in the $\mathrm{AL}$ slow fibers and the SOL in both slow and fast fibers. The EDL and PL showed less reduction in fiber sizes, and the GM was affected only in slow fibers. Fiber-type composition changes were most evident in the muscles with the highest proportion of slow fibers. There was an increase in the percentage of fast oxidative fibers in the AL by $130 \%$, in the SOL ( $28 \%$ ), and in the deep part of the GM by $16 \%$. However, the EDL, PL, and the exterior part of the GM remained unchanged. Also, the myofibrillar ATPase activity showed a significant increase in the SOL muscles, but the EDL muscle groups were similar to controls. Lastly, the SHD activity in SOL, $\mathrm{AL}, \mathrm{PL}$, and $\mathrm{MG}$ muscles remained the same, while it increased in the fast-contracting fibers of EDL. The GPD activity in the SOL and AL increased significantly, while the PL and GM did not show changes, and the EDL showed elevated GPD in the slow-contracting fibers. 
Examining a much longer duration of spaceflight, Sandona et al. (2012) conducted a study in which the SOL and EDL muscles of one mouse flown for 91 days were analyzed for degree of atrophy, fiber type, and gene expression. While no significant degree of atrophy was observed in the EDL of the space-flown mouse, the CSA of SOL fibers was $35 \%$ less in all fiber types, relative to the control groups. While no significant change in the MHC protein bands in EDL was found, the SOL exhibited a reduction of type I MHC and an increase of type IIA and IIB, highlighting the SOL undergoing a slow-to-fast phenotype transition. Along with the observed phenotype transition, down-regulation of the $\mathrm{Ca}^{2+}$-activated $\mathrm{K}^{+}$channels were observed in the SOL. Expression of genes related to stress was found to be upregulated in the case of the EDL but not the SOL. The authors suggested that the differentiated gene expression of the EDL versus the SOL of the spaceflight mouse, combined with the SOL's greater atrophy as compared to the EDL, together suggest that the EDL in $\mu \mathrm{G}$ is activating compensatory pathways that have the effect of protecting the muscle.

Adding dimension, Gambara et al. (2017) investigated for the first time the global gene expression response to 30 days of $\mu \mathrm{G}$ exposure in mouse SOL and EDL muscles. Results showed a reduction of CSA in all fiber types in SOL of flight mice, compared with controls, and a shift toward "fast" type II fibers in both SOL and EDL. Analysis of gene expression found that, in the experimental mice, the primarily slow-phenotype SOL muscle displayed as many as 680 differentially expressed genes. In comparison, the primarily fastphenotype EDL muscle displayed only 72 differentially expressed genes as compared to the control groups. This finding underlines the muscle-specificity of the microgravity-induced transcriptome rearrangement in different muscle types from the hindlimbs of spaceflight mice.

In a complementary study examining many different muscle properties, Tascher et al. (2017) investigated the effect of 30 days spaceflight on molecular pathways in the SOL, EDL, and VL muscles of male mice. Post-flight, the greatest changes were observed in the SOL, beyond those found in the EDL and VL. Results demonstrated that decreases in muscle size and performance occurred as a result of changes in protein balance, mitochondrial function, metabolism, structure, fiber types, calcium signaling, and telomere maintenance. The researchers suggested that decreased protein synthesis, mTOR pathway, myogenesis, oxidative stress, and insulin resistance were possible causes leading to muscle atrophy.

Relatively few studies have been conducted on primate animals. Primate studies have used smaller numbers of subjects than the aforementioned rodent studies, and no relevant primate studies have been conducted in the past 15 years. Nevertheless, two notable primate studies may be mentioned here: Bodine-Fowler et al. (1992) examined the effects of spaceflight on the size and metabolic properties of SOL, TA, and GM muscle fibers of two monkeys after 14 days of spaceflight, as compared with four ground-based control monkeys. Results revealed little to no effect on fiber size in the SOL and GM muscles following spaceflight. The TA muscle fibers, however, appeared to have decreased slightly. Additionally, the SDH activity of the GM muscle fibers of flight monkeys decreased in response to $\mu \mathrm{G}$, with no such change noted in the control group. In sum, the study did not identify any measurable effects of spaceflight on the SOL or TA muscle fibers. On the other hand, in Shenkman et al. (2003), the two spaceflight monkeys flown for 14 days showed a decrease in size of type I fibers of about $30 \%$, while type II fibers decreased by $33 \%$ compared with the pre-flight conditions and to the control groups. The study found that post-flight loss of protein within muscle fibers was a more significant factor in SOL atrophy than the loss of other cellular components, including water. In both spaceflight monkeys, the number of capillaries per SOL fiber was shown to decrease.

Over the past 40 years, a robust corpus of research has documented the significant effects of $\mu \mathrm{G}$ on the skeletal muscles of animals. The research broadly supports the finding that $\mu \mathrm{G}$ negatively impacts skeletal muscle and that these impacts are observed more severely in muscles that counter gravity, such as the VI and SOL, and observed less severely, or in some studies not at all, in muscles such as the EDL and the diaphragm. Likewise, the studies have noted that these muscles' slow-twitch fibers are affected more severely than their fast-twitch fibers. Also, the white regions in these muscles are generally affected more severely when compared to their red counterparts.

Adaptation of the skeletal system to microgravity in humans

In examining changes to bone parameters in humans undergoing spaceflight, some studies have relied 
on indirect measurements, including serum levels of absorption and resorption markers like calcium, vitamin $\mathrm{D}$, osteocalcin, and other minerals, enzymes, proteins, and hormones. Amongst the early metabolic studies in space, Whedon et al. (1971) observed an increase in urinary calcium starting the second week onboard the Gemini- 7 by $23 \%$ and $9 \%$ for the two astronauts. On the contrary, Rambaut et al. (1979) showed that urinary calcium began to increase as soon as one day after weightlessness. With a more comprehensive battery of measurements, Smith et al. (1999) demonstrated that for 115 days in spaceflight, urinary calcium excretion, and bone resorption increased by $50 \%$. Astronauts lost approximately $250 \mathrm{mg}$ of bone calcium per day during flight and recovered this loss at a slower rate of $\sim 100 \mathrm{mg} /$ day for three months post-flight. Similarly, Smith et al. (2005) confirmed that during four to six months of spaceflight, bone resorption markers were markedly greater than pre-flight. Intestinal calcium absorption was significantly lower during flight compared with pre-flight values. Bone calcium balance was significantly more negative during flight than before flight. These findings indicated that spaceflight-induced bone loss is caused by increased bone resorption and decreased intestinal calcium absorption.

Early X-ray densitometry studies magnified the concern of spaceflight-induced loss of bone mineral density (BMD). These studies often compared weightbearing with non-weight bearing bones and can roughly be divided by their focus, either on distal limb bones (forearm, tibia, ankle) and proximal bones (hip, spine, femur). Beginning with distal limb studies, Mack et al. (1967) examined changes in hand and foot bone mass of astronauts aboard the Gemini 4, 5, and 7 missions. The data indicate that spaceflight leads to bone mass loss, though no p-values were reported. The astronauts on Gemini 7 (14-day spaceflight) experienced the least overall loss, whereas astronauts on Gemini 5 (eight-day spaceflight) experienced the most overall bone mass loss. This data also suggests that other variables, besides length of spaceflight, determined the amount of bone loss. A statistically significant coefficient of correlation was reported between calcium intake and bone loss in each astronaut during their respective spaceflight. In a comparison between astronauts from various missions and bed rest results, Vogel et al. (1975), measured the bone density changes of the astronauts in the central left calcaneus (CAL), distal right RAD, and distal right ulna. Results revealed that trabecular and weight-bearing bones, as opposed to cortical and non-weight-bearing bones, are more susceptible to the harmful effects of spaceflight.

Using more advanced technologies such as QCT and ultrasound, Collet et al. (1997) looked at the BMD and bone metabolism in two cosmonauts before and after one-month and six-months in the MIR station. The measurements were performed on the distal (for trabecular bone) and proximal (for cortical bone) nondominant RAD (non-weight bearing) and tibia (weight-bearing). After one month, a decrease in trabecular bone mass was found in the tibia $(2.27 \%)$, whereas no change was observed in the RAD or the cortex of either bone. The CAL was significantly reduced $(7.74 \%)$. After six months, there was a loss of trabecular $(4.5 \%)$ and cortical $(2.9 \%)$ bone of the tibia. Likewise, Vico et al. (2000), measured BMD of cosmonauts at the distal RAD and tibia aboard the MIR space station for either one, two, or six months. Consistent with the previous findings, cosmonauts showed reduced BMD values in tibial trabecular bone and cortical bone. The one-month spaceflight group experienced a decrease of $1.7 \%$ in the trabecular BMD of the tibia. The two-month spaceflight group experienced a $1.6 \%$ reduction in the cortical BMD of the tibia. For the six-month group, cosmonauts showed reduced BMD values $(1.8 \%)$ in tibial cortical bone. The trabecular and cortical bone of the RAD remained unchanged throughout the spaceflights. The researchers postulated that since RAD may be used for locomotion in space, the functions of lower and upper limbs are distorted. Therefore, a new calcium balance may have been established. These findings confirmed rapid bone loss in weight-bearing bones is more pronounced in the trabecular compartment than the cortex. The follow-up data showed incomplete BMD recovery after re-ambulation for most cosmonauts.

As for studies focusing on proximal bone sites, Oganov et al. (1991) measured mineral density of lumbar vertebral spongy tissue in cosmonauts before and after various flight durations. The overall QCT results showed minimal loss of vertebral body trabecular vBMD, $8 \%$ decrease in the posterior elements of the vertebral, and up to $4 \%$ declines in the volume of the adjoining paraspinal muscles. It is noteworthy that the posterior element of the vertebral body serves as muscle attachment points. LeBlanc et al. (2000) reported changes in bone mass tissue from 18 cosmonauts on 12 prolonged missions to the Mir space station. The aBMD results indicated a significant difference in the reduction of bone mineral 
and lean tissue between the whole body and the spine, but not a significant difference between the neck of FEM, spine, trochanter, and pelvis. Moreover, the bone loss rate for two of the crew members who stayed in space for 311 and 438 days, respectively, was $50 \%$ compared to the other cosmonauts. These findings suggest that the in-flight exercise countermeasure was inadequate as participants experienced bone loss despite adhering to the Russian exercise regimen (Table 7).

Expanding to include both spine and hip, Lang et al. (2004) showed that astronauts who spent four-six months aboard the ISS suffer from considerable loss of trabecular and cortical bone in the hip and smaller losses in the spine. For the spine, bone loss occurred at an average rate of $0.8 \%$ to $0.9 \%$ per month. In contrast with Oganov et al. (1991), this finding did not observe a focal loss of bone in the posterior spinal elements; the posterior elements underwent similar changes as the vertebral bodies. The researchers suggested that this surprising finding was possibly caused by the newly developed exercise device designed to maintain the strength of the lower back muscles. The greatest losses occurred in the hip at rates of 1.2 to $1.5 \%$ per month. Differential patterns of cortical and trabecular bone mineral loss at the hip was observed. More than $90 \%$ of the mineral loss was from cortical bone (1.6 to $1.7 \%$ per month), which occurred primarily by cortical thinning. Trabecular vBMD was lost at a rate of 2.2 to $2.7 \%$ per month. The higher percentage loss of trabecular bone in the proximal femur was consistent with previous findings, which showed loss of trabecular bone at distal femoral and tibial sites. Vico et al. (2017) further explored changes in the cortical and trabecular bone of the lumbar spine, hip, and femoral neck. BMD of the hip (total and femoral neck) and lumbar spine (L1-L4) decreased at a rate of about $1 \%$ per month. At the tibia, the cortical and trabecular densities and cortical thickness decreased, while cortical porosity increased by $15 \%$. These numbers were smaller than those observed on the Mir space station, probably due to the use of ARED and robust exercise programs on the ISS. Bone resorption was significantly increased during spaceflight. Bone markers reflecting osteocyte activity and periosteal metabolism (sclerostin, periostin) levels did not change significantly. The authors concluded that some changes to bone from spaceflight may be partly irreversible.

Finally, utilizing the subject-specific finite element (FE) models, Keyak et al. (2009) studied proximal femoral strength in 13 astronauts under two loading condition models: a single-limb stance and a fall onto the posterolateral aspect of the greater trochanter. Results revealed that proximal femoral strength (Ffe) under stance loading was reduced in all participants. When normalized for stance loading during the flight period, the Ffe demonstrated a drop at a mean rate of $2.6 \%$ per month. For the fall loading, it showed a decrease of $2 \%$ per month. The authors suggested that significant reductions of proximal femoral strength may lead to an enhanced risk for hip fractures as astronauts age, especially several decades following their space travels.

Adaptation of the skeletal system to microgravity in animals

Effects on the skeletal system in animals varied according to site, but some results, especially from earlier studies, are reported with less specificity. During the Soviet biosatellite Cosmos-936, Gurovsky et al. (1980) investigated the whole-body effects on male rats of prolonged weightlessness (18.5-day flight). The researchers observed a delay in weight gain, osteoporosis, bone density, and mineral content loss, and a $30 \%$ decrease of the mechanical bending strength in the femoral and tibial bones among the weightless rats compared to controls (significance levels unstated). Backup et al. (1994) conducted two distinct experiments to examine the effects of short-term spaceflight on gene expression in bone in growing rats. The dynamic bone histomorphometry results indicated that the effects of spaceflight on the musculoskeletal system include decreased expression of some bone-specific genes, as well as decreased bone formation. To further elucidate the bone formation and resorption effects, Morey et al. (1978) examined 63 male rats flown for 19.5 days aboard Cosmos 782 biological satellite. Results showed that during flight, bone formation rate decreased about $47 \%$ compared to control groups. This dramatic decrease occurred along with an arrest line at both the periosteum and the endosteum, indicating that bone growth completely stopped in flight animals. Findings suggest that bone formation started again three days post-flight. Cann et al. (1983) likewise aimed to measure the effect of spaceflight on bone resorption in growing rats for 20 days spaceflight of COSMOS 1129. The results revealed that bone resorption in rats did not significantly change at the end of the flight periods, while mineral excretion increased during flight, four to five times that of control values. The overall results show that mineral homeostasis 
did not adapt to the $\mu \mathrm{G}$ exposure of this experiment.

Moving on to specific bone sites in the rostralto-caudal direction, Macaulay et al. (2017) investigated structural and molecular changes in mice calvariae during a 30-day spaceflight. The Micro-computed Tomography $(\mu \mathrm{CT})$ results demonstrated that in spaceflight mice, calvaria bone structure was not significantly different from that of their controls. Sclerostin, the secreted glycoprotein of the Sost gene, is a potent inhibitor of bone formation. Quantitative PCR results showed that Sost mRNA expression was 16-fold greater in the spaceflight group than in the control group. Therefore, the researchers concluded that bone formation might have been suppressed in mice exposed to spaceflight. This study suggested that calvaria mechanotransduction pathways may be altered during spaceflight, which could reflect vascular and interstitial fluid adaptations in nonweight-bearing bones. Dagdeviren et al. (2018) likewise investigated non-weight-bearing bones in the head of female mice flown for 13 days, as well as male mice flown on for 30 days, with flight habitat and vivarium control groups. $\mu \mathrm{CT}$ analysis of mandibular bone demonstrated a significant decrease in $\mathrm{BV}$ in the 30-day flight and habitat control mice, as well as a significant increase in BV in the 13-day flight and habitat control mice, as compared with their vivarium groups. Tissue density of mandibular bone in the STS-135 flight and its habitat control was significantly higher than in the vivarium group. This study provides further data that spaceflight may affect non-weight-bearing bones, although interpretation of the findings is limited by various factors. Ghosh et al. (2016) further studied spaceflight effects on the mandible in mice flown for 15 days on the STS-131 Space Shuttle mission and 13 days on the STS-135 mission. The findings demonstrate that minerals are redistributed within the skeleton. The researchers suggested that in addition to spaceflight-induced cephalic fluid shifts, other factors associated with spaceflight such as elevated $\mathrm{CO}_{2}$ levels and vascular adaptations may also be important factors in the remodeling process of non-weight-bearing bones in the head.

Studying the spine, Zernicke et al. (1990) tested the biomechanical and biochemical responses of lumbar vertebral bodies of rats during the Cosmos 1887 biosatellite flight. Results showed that the average mass of flight rat L6 vertebrae was smaller than in the control groups. Compressional stiffness, initial maximum load, and linear load of the flight vertebral bodies were also lower relative to control groups. The researchers suggested that the lack of strength and stiffness and smaller amounts of hydroxypyridinone indicated that the 12.5-day spaceflight may have detrimental effects on bone maturation of trabecular properties in vertebral bodies. For the first time, Zerath et al. (1996) studied bone mineralization effects of spaceflight on the pelvis of two male monkeys using noninvasive methods. The results showed that 11.5 days of spaceflight-induced a significant bone loss in the iliac trabecular area, associated with altered mineralizing activity in the two young male rhesus monkeys.

Comparing changes in the tibia and humerus, Jee et al. (1983) aimed to ascertain changes in trabecular bone formation that occurred in rats during 18.5 days of spaceflight. The results demonstrated that the flight rats had a significantly reduced mass of mineralized tissue in tibia, relative to controls. The flight rats also exhibited lower trabecular bone volumes and increased fat content at skeletal sites. The osteoblast population in the proximal humeral metaphysis of flight rats was partially diminished near the growth plate. Moreover, a decrease in the number of osteoblasts at the growth cartilage metaphyseal junction was observed among experimental rats as compared to controls, while osteoclasts had no significant changes. In Vico et al. (1988), histomorphometric measurements of proximal tibial metaphysis showed that trabecular bone volume, thickness, and density were significantly reduced, while trabecular separation was higher in in-flight rats. No significant changes in the proximal FEM metaphysis were observed in the flight group. At the cellular level, resorption activity in tibia was not affected by spaceflight, while osteoid surfaces were significantly decreased in flight rats. In the FEM, osteoclast and osteoid parameters were not significantly altered. Looking specifically at the FEM, Mechanic et al. (1990) found that after 12.5 days of spaceflight, mineralization in the distal half of the diaphysis decreased, while levels in the proximal sections were closer to levels in the control groups. Thus, the defect in mineralization, which is associated with spaceflight and landing, was concluded to be most severe in the distal section of the diaphysis.

To study ankle bones, Gerbaix et al. (2018) analyzed the structure and density changes of the CAL, navicular, and talus of mature mice after 30 days of spaceflight. Results showed that CAL Bone Volume Fraction (BV/TV) decreased $(-6.4 \%)$ in the flight group, as did the cortical thickness $(-11.69 \%)$, relative to the control group. Spaceflight-induced bone loss in all bones was site- and compartment-specific, while the tissue 
mineral density (TMD) of the remaining bone did not change. 3D bone reconstruction analysis showed cortical thinning and bone erosion, mainly at tendon-bone junction and ground contact bone sites. Muscle atrophy at reduced muscle insertions were observed to significantly impact the structure of the tendon-bone joint in terms of cortical thinning. The loss of ground reaction forces and muscular load in microgravity may explain the reduction in cortical thickness at the point of ground contact bone sites and muscle insertions.

Demonstrating the importance of connective tissues, Fitzgerald et al. (2019) investigated degradation of the articular cartilage (AC) and sternal cartilage (SC) of male mice in space for 30 days. Histological analysis revealed that spaceflight $\mathrm{AC}$ samples, particularly the femoral condyle, degraded at the tissue level with less proteoglycan compared to ground controls. There was also a reduction in mRNA expression of mechanoresponsive and structural cartilage matrix proteins compared to controls. Osteophyte formation was observed in three spaceflight samples as well as in two control samples. spaceflight mice had significantly higher signs of overall cartilage degradation than controls. Weight-bearing $\mathrm{AC}$ was found to be negatively affected by the unloading of $\mu \mathrm{G}$. The researchers suggested that maintenance of physiological loading on AC during spaceflight would minimize AC damage.

Effectiveness of exercise countermeasure on the musculoskeletal system

\section{Skeletal muscle adaptations}

Data on the effectiveness of exercise countermeasures in preserving muscle structure and function in spaceflight have been limited. Most of the exercise interventions presented in this section have proven either inadequate or partially effective. Likewise, exercise prescriptions used in preventing muscle size and strength attenuation have shown success to varying degrees. Thornton et al. (Thornton 1977) evaluated arm and leg strength from Skylab missions 2, 3, and 4. As for daily exercise duration, there were increases from Skylab 2 to 3 to 4 . In terms of percentage decrease of body mass and arm and leg strength, Skylab 2 resulted in the highest decreases, and Skylab 4 resulted in the lowest decreases. Changes in leg extension forces from pre- to post-flight were $-0.89 \% /$ day, $-0.44 \% /$ day, and $-0.09 \% /$ day in Skylabs 2, 3, and 4, respectively. The same trend is found for changes in lean body mass, with $-0.089 \%$ day,
$-0.019 \% /$ day, $-0.011 \% /$ day in Skylabs 2, 3, and 4, respectively, as well as with measurements in total body weight with $-0.13 \% /$ day, $-0.08 \% /$ day, $-0.02 \% /$ day in Skylabs 2, 3, and 4, respectively. The increase in daily exercise duration, as well as the addition of the treadmill device, which targeted the legs, clearly reduced the loss of muscle volume and strength, as well as body mass.

Furthermore, Trappe et al. (2009) provided a detailed overview of the in-flight exercise program performed by nine crew members while on the ISS for six months. The exercise equipment used included the Treadmill with Vibration Isolation System (TVIS), Cycle Ergometer with Vibration Isolation System (CEVIS), and the Interim Resistive Exercise Device (iRED). Results revealed that the average muscle mass loss (13\%) was slightly less than the previous reports (17\%) (LeBlanc et al. 2000). MVC decreased $14 \% \pm 2 \%$, while measurements of force-velocity in isokinetic tests decreased by $20 \%$ to $29 \%$ on average. Fiber type distributions in the GAS, measured by MHC isoforms, showed a $12 \%$ decrease in MHC I, with a $4 \%$ increase in hybrid MHC I/IIa fibers and a $9 \%$ increase in $\mathrm{MHC}$ IIa fibers. The SOL showed a $17 \%$ decrease in MHC I fibers, with a $12 \%$ increase in hybrid fiber types. The exercise program performed on the ISS was mainly of high volume and moderate intensity. Findings indicated that this exercise program was beneficial but did not completely protect the volume and mechanical function of the participants' calf muscles, deeming this program inadequate. In a later study, Fitts et al. (2010) also confirmed that the exercise countermeasures employed during ISS increments 5 to 11 were incapable of providing the intensity and wide range of motion needed to prevent muscle atrophy.

Gopalakrishnan et al. (2010) directly measured the loading during in-flight exercise countermeasures and showed that, despite the exercise program, muscle atrophy and reductions in muscle strength still occurred. Results showed a general loss across all lower extremity muscle groups in concentric (isometric and isokinetic) strength. The isometric strength decreased by approximately $2 \%$ in the hip abductors, and about $35 \%$ in the hip adductors. Isokinetic concentric changes occurred from a $2 \%$ increase in hip extensor strength to a $24 \%$ decrease in the knee flexors. The post-flight peak isokinetic torque was 2.3 times greater in the knee flexors compared to the knee extensors. Foot force measurements showed that despite the use of in-flight exercise countermeasures, these physical training in terms of 
volume and intensity were insufficient to replicate the daily mechanical loading experienced on Earth.

Fitts et al. (2013) were first to evaluate the effects of weightlessness plus exercise countermeasures on anaerobic and aerobic enzyme activity, and the content of glycogen and lipids in slow and fast fibers of the SOL and GAS muscles. The study was conducted on two groups performing two different exercise programs. One group of about four subjects exercised with a treadmill for more than 200 minutes per week (HT), while the other group consisting of about three subjects ran less than 100 minutes per week (LT). Post-flight results showed that the treadmill running partially protected against fiber atrophy. It also helped individuals in the HT group to increase muscle aerobic enzyme activity. Overall, this study showed that enzyme capacity could be maintained by performing adequate treadmill running that exceeded $200 \mathrm{~min} / \mathrm{wk}$. Thus, the muscle weakness that occurs after prolonged spaceflight is not a direct result of a loss of enzyme capacity and muscle substrates.

Since the iRED failed to prevent bone and muscle loss, the ARED was designed and deployed on the ISS during Expedition 18 in response to several limitations of the iRED, and to improve the overall effectiveness of the exercise countermeasures. In a retrospective study, English et al. (2015) measured changes in isokinetic strength in 37 ISS crew members, comparing gender differences, differences between iRED vs. ARED, and correlations between pre-flight strength and strength list during flight. For gender differences, strength losses were found to be approximately $5 \%$ to $8 \%$ greater in female subjects than in males. Comparing equipment, strength reductions were greater in the earlier iRED missions than in the later ARED missions, but the differences were not significant. Moderate correlations were found between higher strength before launch and strength reduction percentage after landing. The use of neither the iRED nor ARED was able to prevent isokinetic strength loss; however, these results suggest that exercise involving the ARED might be better suited to prevent losses in strength compared to exercise involving the iRED.

Concerning the effectiveness of exercise countermeasure on the trunk muscles, Hides et al. (2016) reported the case results of one adult male astronaut while onboard for six months. The crewmember performed an individualized exercise program consisting of resistive and aerobic training using ARED and a treadmill. Post-flight measurements showed no significant difference in MF CSA at the L2 level as well as the L3 level; for each of these two vertebral levels, the size of the MF increased by $7 \%$ as compared with the pre-flight measurement. The MF at the L4 level showed a $1 \%$ change, and at the L5 level the CSA of the MF decreased by $29 \%$ as measured at the lumbrosacral junction. The transversus abdominis (TrA) muscle was observed to respond to $\mu \mathrm{G}$ by decreasing in thickness, whereas the internal oblique muscle increased in thickness. Findings revealed that pre-and post-flight interventions proved somewhat effective in preventing trunk muscle mass reduction and restoring balance, respectively.

Furthermore, Burkhart et al. (2019) aimed to discover a correlation between the in-flight exercise program and attenuation changes of the paraspinal muscles during four- to seven-month spaceflight missions. On average, the subjects spent $98 \pm 41$ minutes exercising on a treadmill per week, $89 \pm 52$ minutes exercising on a cycle ergometer per week and participated in $4.5 \pm 1.2$ sessions of resistance training per week. The changes in the CSA of the paraspinal muscle for this study were previously reported in this paper. Overall findings indicated that the in-flight resistance exercise sessions showed effectiveness in muscle mass loss prevention of the spinal extensors (ES and MF), whereas cycle ergometer exercise helped prevent QL CSA loss. No correlations between exercise volume and change in paraspinal muscle attenuation was observed.

On the other hand, McNamara et al. (2019) analyzed the CT scans of crewmembers in the iRED era. They showed that although the paraspinal muscles were still experiencing atrophy in space, the use of iRED preserved lumbopelvic muscle volume. Crewmembers who used iRED twice a week had $11 \%$ decrease in lumbopelvic volume, whereas those who used iRED six times a week experienced only a $2 \%$ decrease in lumbopelvic volume. Crewmembers who spent more time on CEVIS had higher decreases in muscle attenuation. On the other hand, the psoas muscle, as a hip flexor, did not show significant levels of degradation. Both iRED and ARED need exercise protocols that mimic spine loading on Earth. These results demonstrate the need to target muscles responsible for postural stability in microgravity to prevent their degradation while on missions.

Lastly, Koryak (2019) studied an exercise regimen with three modes consisting of bilateral supine leg presses and arm presses. The first mode consisted of $70 \%$ force-velocity exercise, and $15 \%$ velocity- and $15 \%$ force-requiring exercises. The second mode mainly 
consisted of a velocity required exercise $(70 \%)$, with the other two exercise types at $15 \%$. The third mode primarily consisted of a force exercise $(70 \%)$. This threeday cycle was repeated twice over six days, with the seventh day as rest, for 16 weeks. The researchers confirmed that the exercise regimen employed and the exercise devices available on the ISS were not adequate to simulate exercise on Earth and, thus, was not able to prevent muscle atrophy or weakness in TS muscles.

\section{Skeletal system adaptations}

LeBlanc et al. (2000) assessed bone loss duringlong duration sojourns (188 days on average) to the Mir space station. They revealed that, despite the use of extensive exercise programs onboard (Table 7), a significant reduction of bone mineral was observed in the spine.

Using Capacitance-based insoles, Cavanagh et al. (2010) compared average foot-forces during exercise to the amount of bone mass lost in lower extremity (LE) during long-duration stays on the ISS. In-shoe forces during walking and running exercises on the TVIS were reduced by $25 \%$ and $46 \%$, respectively, relative to the values on Earth. Regarding the loading of single foot forces during resistive exercise on iRED, measures varied from 0.2 to $1.3 \mathrm{BW}$ during "bent over row" and "single-leg heel raise." Limited cycling results indicated that LE loading on CEVIS ergometer is significantly low. Of note is that, while total exercise time was 146.8 minutes per day on average, total time under loaded tension was $\sim 43$ minutes. The per-month reduction in BMD was $0.71 \%$ and $0.83 \%$ in the femoral neck and lumbar spine, respectively. Based on these findings, the exercise duration and/or loading applied to the LE were inadequate to prevent reductions in BMD.

In another prominent study, Smith et al. (2012) compared the performance of the iRED and ARED as countermeasures for bone loss. Subjects were divided into two groups (ARED vs iRED). Both groups had the same exercise prescription (Table 7). Results showed a significant interaction between spaceflight duration and use of iRED vs. ARED in BMC and BMD of the pelvis, right femoral neck, and total left hip. Interactions were found in trochanter BMD of both sides and right hip BMD. Similar interactions of flight duration and exercise device were found in body fat and lean muscle mass percentage. For the ARED group, BMD in most measured areas (except lumbar spine) was not significantly different from pre-flight. Protein and energy intake were also measured to significantly correlate with less BMC loss in the pelvis. This study confirmed for the first time that improvements in nutrition and resistance exercise regimens reduce the BMD deficits in prolonged missions.

Later, Smith et al. (2015) evaluated different types of countermeasures to resist bone loss in space. The astronauts were categorized into three groups: one group had access to the iRED, another group had access to the ARED, and the last group had access to the ARED and regularly consumed bisphosphonate alendronate (Bis+ARED). By day 120, bone formation was significantly higher in ARED crew members than it was before flight, assessed by bone sample alkaline phosphatase (BSAP), total alkaline phosphatase, and/or osteocalcin circulating concentrations. BSAP increased by almost $60 \%$ by day 180 in the ARED group and about $25 \%$ in the iRED group, but it was about $11 \%$ lower than pre-flight levels in Bis+ARED subjects. Bone resorption increased in ARED and iRED groups but was largely unchanged in Bis + ARED crew members. Serum calcium was significantly lower in Bis+ARED subjects than in other groups before and throughout the flight. Urinary calcium excretion was generally greater during flight for both ARED and iRED groups. These results shed light on the previous findings that bone loss can be mitigated through exercise-induced increased bone formation, and pharmacological agents like bisphosphonates.

\section{Conclusions}

This is the first study to 1) systematically identify, evaluate, and synthesize the heretofore disparate scientific literature regarding the effects of direct exposure to $\mu \mathrm{G}$ on the musculoskeletal system, taking into account for the first time both bone and muscle systems of both humans and animals; and to 2) investigate the efficacy and limitations of exercise countermeasures on the musculoskeletal system under $\mu \mathrm{G}$ in humans.

This study led to several significant findings:

\section{Musculoskeletal response}

- The research demonstrates that the musculoskeletal adaptations to the space environment are both site-specific and compartment specific.

- Effects of spaceflight on human skeletal muscle have been found to vary substantially by site, trending toward increased effects in the inferior direction, 
e.g., the lower spine and legs. This could reflect partial mitigation of upper-limb atrophy due to using the upper limbs for locomotion in $\mu \mathrm{G}$.

- The research on animal skeletal muscles supports the finding that $\mu \mathrm{G}$ negatively impacts the skeletal muscle system more severely in muscles which counter gravity, such as the VI and SOL.

- Animal studies have shown that slow-twitch fibers are affected more severely than fast-twitch fibers.

- The site-specificity and compartmentspecificity in skeletons are also evident from losses being more severe in weight-bearing bones such as the tibia, particularly in the trabecula.

- Muscle atrophy at attachments may significantly impact the structure of the tendon-bone connection, in terms of cortical thinning.

- The loss of ground reaction forces and muscular load in $\mu \mathrm{G}$ may explain the reduction in cortical thickness at the point of ground-contact bone sites and muscle attachments.

\section{Exercise countermeasures}

- Exercise, particularly resistance training, is the cornerstone of countermeasures to prevent microgravityinduced remodeling of musculoskeletal systems.

- Exercise prescriptions used in preventing the attenuation of muscle size and strength, as well as BMD, have shown success to varying degrees.

- Accordingly, none of the exercise interventions presented in this study were found fully effective in preserving muscle structure and function or BMD in spaceflight conditions.

- NASA's first-generation resistance exercise device, iRED, was not able to elicit loads comparable to exercise on Earth; therefore, it failed to maintain muscle and bone mass during long-duration $\mathrm{SF}$.

- The newer resistive exercise device, ARED, along with more robust physical training programs, have been partially effective in attenuating bone and muscle loss during prolonged space missions, leading to the conclusion that optimizing exercise regimens on existing equipment will yield better results at this time than development of new exercise equipment.

- Studies confirmed that bone loss could be mitigated through exercise-induced increased bone formation in combination with pharmacological agents like bisphosphonates.

\section{Limitations}

This review is limited to musculoskeletal outcomes and exercise countermeasures. Therefore, other physiological systems and interventions were not considered. Similarly, this study focuses only on the effects of direct exposure to $\mu \mathrm{G}$; thus, ground-based models were not included, although such studies have also shown efficacy of exercise as a countermeasure to musculoskeletal and cardiovascular atrophy and thus may provide further insights to improve prescribed exercise regimens. ( $\mathrm{Li}$ et al. 2017, Luciano et al. 2017) Heterogeneity in the study designs, methodologies, and outcomes made meta-analysis impossible, so we presented a narrative synthesis of the results. Limitations were found in the designs of spaceflight experiments. For example, investigations are inherently limited to the small sample sizes and varied flight durations in the majority of the human spaceflight studies. Randomized sampling methods and blinding of the participants and assessors cannot feasibly be employed. Technological advancements caused variability in assessment methods and outcomes. For instance, throughout the past 50 years, various BMD-measuring tools have been utilized, such as single-photon absorptiometry, DXA, and QCT. The crewmembers are mainly middle-aged males, thus the physiological adaptations between different sexes and ages are not considered. Astronauts are generally extraordinarily fit and healthy individuals put into an extreme environment; this population represents a limited range of human body types and health statuses. At the same time, inherent variability exists in human participants' age, sex, nutritional status, exercise levels, and flight durations, and thus the human results should be interpreted with caution. Further, there are possible unpublished studies on the topic. This systematic review is, therefore, prone to evidence selection bias from unpublished studies.

According to our research results, many spaceflight experiments consist of pre-posttest design without control arms, or with ground-based control groups. Therefore, it is difficult to rule out the effect of other variables such as galactic rays, temperature, accelerations, and high vibration forces associated with take-off and landing. Some of the space missions were dedicated to the study of life sciences, which incorporated human physiology testing. Because astronauts have been participating in in-flight exercise programs, the reported outcomes are not of spaceflight alone per se, but rather of 
space flight plus the exercise conditions that crew members were subjected to while in space. It is worth mentioning that in some cases, the in-flight functional testing itself may have worked as a countermeasure and affected the results being measured. While in-flight assessments of physiological adaptations are beneficial, it is crucial to employ technologies and research methods that reduce the observer effect. In older studies, individuals performed ad libitum in-flight exercises, which were not closely documented. In other cases, the in-flight exercise programs had a large disparity in volume and mode of activity among the crewmembers, which makes it difficult to assess precisely the effectiveness of the exercise programs. The in-flight activities of the astronauts are not documented in these studies. This is relevant because each crewmember has different responsibilities throughout the mission, thus their levels of physical activity are different. Many of the exercise analyses are based on self-report of in-flight exercises. This lack of detailed information about the intensity, volume, and duration of in-flight exercise complicates the precise explanation of exercise's impact on musculoskeletal changes in the $\mu \mathrm{G}$ environment. This assortment limited the comparability of the findings and affected the quality assessment process.

\section{Suggestions for future research}

The relatively large number of studies on animal muscle allows us to see the nuances in these general findings. Several recent studies have begun to point toward identifying the specifics of underlying mechanisms and processes. Still, more work remains to be done in that area, mainly because all studies provide only data from pre-flight, post-flight, and sometimes post-recovery measurements, yet, no measurements have been performed during the interval of spaceflight itself. Significant research has been conducted focusing on the hindlimbs of rats or mice. Relatively few studies have been conducted using nonhuman primate animals, primate studies have used smaller numbers of subjects than the aforementioned rodent studies, and no significant primate studies have been conducted in the past 15 years. Given the structural complexity of the musculoskeletal system, including bone and muscle as well as tendons, ligaments, cartilage, and other connective tissues, and given the anatomical and functional interconnectedness of these parts, bone loss is directly affected by muscle atrophy as well as by changes in muscle strength. Due to this dynamic complexity, the reductionist approach, which focuses on each part separately, has answered some questions but left many unanswered. The interplay between bone, muscle, and cartilage needs to be given full recognition and consideration in future research. The musculoskeletal adaptations in a $\mu \mathrm{G}$ environment merit an in-depth investigation beyond bone and muscle.

\section{Conflict of Interest}

There is no conflict of interest.

\section{Acknowledgements}

The authors received no funding for this work.

\section{Abbreviations}

Adductor Longus, AL; Advanced Resistive Exercise Device, ARED; Bodyweight, BW; Bone Mineral Content, BMC; Bone Mineral Density, BMD; Calcaneus, CAL; Control, CON; Cross-Sectional-Area, CSA; Cycle Ergometer with Vibration; Isolation and Stabilization, CEVIS; Extensor Digitorum Longus, EDL; Electromyographic, EMG; Electromechanical delay, EMD; Erector Spinae, ES; Femur, FEM; Gastrocnemius Lateralis, GL; Gastrocnemius Medialis, GM; Gastrocnemius Muscle, GAS; Ground-based control, GC; Hindlimb Suspension, HLS; Interim Resistive Exercise Device, iRED; International Space Station, ISS; Magnetic Resonance Imaging, MRI; Micro-computed Tomography, $\mu \mathrm{CT}$; Microgravity, $\mu \mathrm{G}$; Multifidus, MF; Myosin Heavy Chain, MHC; Plantaris, PL; Psoas, PS; Quadriceps, Quad; Quadratus Lumborum, QL; Radius, RAD; Rectus Femoris, RF; Soleus, SOL; Spaceflight, SF; Succinate Dehydrogenase, SDH; Synchronous, SYNC; Tibialis Anterior, TA; Treadmill with Vibration Isolation and Stabilization, TVIS; Triceps Surae, TS;Vastus Medialis, VM; Vastus Lateralis, VL;Vastus Intermedius, VI; Vivarium, VIV

\section{References}

AKIMA H, KAWAKAMI Y, KUBO K, SEKIGUCHI C, OHSHIMA H, MIYAMOTO A, FUKUNAGA T: Effect of short-duration spaceflight on thigh and leg muscle volume. Med Sci Sports Exerc 32: 1743-1747, 2000. https://doi.org/10.1097/00005768-200010000-00013 
ALLEN DL, YASUI W, TANAKA T, OHIRA Y, NAGAOKA S, SEKIGUCHI C, HINDS WE, ROY RR, EDGERTON VR: Myonuclear number and myosin heavy chain expression in rat soleus single muscle fibers after spaceflight. J Appl Physiol 81: 145-151, 1996. https://doi.org/10.1152/jappl.1996.81.1.145

ANTONUTTO G, BODEM F, ZAMPARO P, DI PRAMPERO PE: Maximal power and EMG of lower limbs after 21 days spaceflight in one astronaut. J Gravit Physiol 5: 63-66, 1998.

ANTONUTTO G, CAPELLI C, GIRARDIS M, ZAMPARO P, DI PRAMPERO PE: Effects of microgravity on muscular explosive power of the lower limbs in humans. Acta Astronaut 36: 473-478, 1995. https://doi.org/10.1016/0094-5765(95)00133-6

ANTONUTTO G, CAPELLI C, GIRARDIS M, ZAMPARO P, DI PRAMPERO PE: Effects of microgravity on maximal power of lower limbs during very short efforts in humans." J Appl Physiol (1985) 86: 85-92, 1999. https://doi.org/10.1152/jappl.1999.86.1.85

ARKSEY H, O'MALLEY L: Scoping studies: towards a methodological framework. Int J Soc Res Methodol 8: 19-32, 2005. https://doi.org/10.1080/1364557032000119616

BACKUP P, WESTERLIND K, HARRIS S, SPELSBERG T, KLINE B, TURNER R: Spaceflight results in reduced mRNA levels for tissue-specific proteins in the musculoskeletal system." Am J Physiol 266: E567-573, 1994. https://doi.org/10.1152/ajpendo.1994.266.4.E567

BAILEY JF, MILLER SL, KHIEU K, O'NEILL CW, HEALEY RM, COUGHLIN DG, SAYSON JV, CHANG DG, HARGENS AR, LOTZ JC: From the international space station to the clinic: how prolonged unloading may disrupt lumbar spine stability. Spine J 18: 7-14, 2018. https://doi.org/10.1016/j.spinee.2017.08.261

BALDWIN KM, HERRICK RE, MCCUE SA: Substrate oxidation capacity in rodent skeletal muscle: effects of exposure to zero gravity. J Appl Physiol (1985) 75: 2466-2470, 1993. https://doi.org/10.1152/jappl.1993.75.6.2466

BELL GJ, MARTIN TP, ILYINA-KAKUEVA EI, OGANOV VS, EDGERTON VR: Altered distribution of mitochondria in rat soleus muscle fibers after spaceflight. J Appl Physiol (1985) 73: 493-497, 1992. https://doi.org/10.1152/jappl.1992.73.2.493

BODINE-FOWLER SC, ROY RR, RUDOLPH W, HAQUE N, KOZLOVSKAYA IB, EDGERTON VR: Spaceflight and growth effects on muscle fibers in the rhesus monkey. J Appl Physiol (1985) 73 (2 Suppl): 82S-89S, 1992. https://doi.org/10.1152/jappl.1992.73.2.S82

BURKHART K, ALLAIRE B, BOUXSEIN ML: Negative effects of long-duration spaceflight on paraspinal muscle morphology. Spine (Phila Pa 1976) 44: 879-886, 2019. https://doi.org/10.1097/BRS.0000000000002959

CAIOZZO VJ, BAKER MJ, HERRICK RE, TAO M, BALDWIN KM: Effect of spaceflight on skeletal muscle: mechanical properties and myosin isoform content of a slow muscle. J Appl Physiol (1985) 76: 1764-1773, 2004. https://doi.org/10.1152/jappl.1994.76.4.1764

CAIOZZO VJ, BAKER MJ, HERRICK RE, TAO M, BALDWIN KM: Microgravity-induced transformations of myosin isoforms and contractile properties of skeletal muscle." J Appl Physiol (1985) 81: 123-132, 1996. https://doi.org/10.1152/jappl.1996.81.1.123

CANN CE, ADACHI RR: Bone resorption and mineral excretion in rats during spaceflight. Am J Physiol 244: R327331, 1983. https://doi.org/10.1152/ajpregu.1983.244.3.R327

CAVANAGH PR, GENC KO, GOPALAKRISHNAN R, KUKLIS MM, MAENDER CC, RICE AJ: Foot forces during typical days on the international space station. J Biomech 43: 2182-2188, 2010. https://doi.org/10.1016/j.jbiomech.2010.03.044

CHANG DG, HEALEY RM, SNYDER AJ, SAYSON JV, MACIAS BR, COUGHLIN DG, BAILEY JF, PARAZYNSKI SE, LOTZ JC, HARGENS AR: Lumbar spine paraspinal muscle and intervertebral disc height changes in astronauts after long-duration spaceflight on the international space station. Spine (Phila Pa 1976) 41: 1917-1924, 2016. https://doi.org/10.1097/BRS.0000000000001873

COLLET P, UEBELHART D, VICO L, MORO L, HARTMANN D, ROTH M, ALEXANDRE C: Effects of 1- and 6-month spaceflight on bone mass and biochemistry in two humans. Bone 20: 547-551, 1997. https://doi.org/10.1016/S8756-3282(97)00052-5 
DAGDEVIREN D, KALAJZIC Z, ADAMS DJ, KALAJZIC I, LURIE A, MEDNIEKS MI, HAND AR: Responses to spaceflight of mouse mandibular bone and teeth. Arch Oral Biol 93: 163-176, 2018. https://doi.org/10.1016/j.archoralbio.2018.06.008

EDGERTON VR, ZHOU MY, OHIRA Y, KLITGAARD H, JIANG B, BELL G, HARRIS B, SALTIN B, GOLLNICK PD, ROY RR, et al: Human fiber size and enzymatic properties after 5 and 11 days of spaceflight. J Appl Physiol (1985) 78(5): 1733-1739, 1995. https://doi.org/10.1152/jappl.1995.78.5.1733

ENGLISH KL, LEE SMC, LOEHR JA, PLOUTZ-SNYDER RJ, PLOUTZ-SNYDER LL: Isokinetic strength changes following long-duration spaceflight on the ISS. Aerosp Med Hum Perform 86(12 Suppl): A68-A77, 2015. https://doi.org/10.3357/AMHP.EC09.2015

ESSER KA, HARDEMAN EC: Changes in contractile protein mRNA accumulation in response to spaceflight. Am J Physiol 268(2 Pt 1): C466-471, 1995. https://doi.org/10.1152/ajpcell.1995.268.2.C466

FITTS RH, COLLOTON PA, TRAPPE SW, COSTILL DL, BAIN JL, RILEY DA: Effects of prolonged space flight on human skeletal muscle enzyme and substrate profiles. J Appl Physiol (1985) 115: 667-679, 2013. https://doi.org/10.1152/japplphysiol.00489.2013

FITTS RH, TRAPPE SW, COSTILL DL, GALLAGHER PM, CREER AC, COLLOTON PA, PETERS JR, ROMATOWSKI JG, BAIN JL, RILEY DA: Prolonged space flight-induced alterations in the structure and function of human skeletal muscle fibres. J Physiol 588 (Pt 18): 3567-3592, 2010. https://doi.org/10.1113/jphysiol.2010.188508

FITZGERALD J, ENDICOTT J, HANSEN U, JANOWITZ C: Articular cartilage and sternal fibrocartilage respond differently to extended microgravity. NPJ Microgravity 5: 3, 2019. https://doi.org/10.1038/s41526-019-0063-6

GAMBARA G, SALANOVA M, CICILIOT S, FURLAN S, GUTSMANN M, SCHIFFL G, UNGETHUEM U, VOLPE P, GUNGA HC, BLOTTNER D: Gene expression profiling in slow-type calf soleus muscle of 30 days space-flown mice. PLoS One 12: e0169314, 2017. https://doi.org/10.1371/journal.pone.0169314

GERBAIX M, WHITE H, COURBON G, SHENKMAN B, GAUQUELIN-KOCH G, VICO L: Eight days of earth reambulation worsen bone loss induced by 1-month spaceflight in the major weight-bearing ankle bones of mature mice. Front Physiol 9: 746, 2018. https://doi.org/10.3389/fphys.2018.00746

GHOSH P, STABLEY JN, BEHNKE BJ, ALLEN MR, DELP MD: Effects of spaceflight on the murine mandible: Possible factors mediating skeletal changes in non-weight bearing bones of the head. Bone 83: 156-161, 2016. https://doi.org/10.1016/j.bone.2015.11.001

GOODSHIP AE, CUNNINGHAM JL, OGANOV V, DARLING J, MILES AW, OWEN GW: Bone loss during long term space flight is prevented by the application of a short term impulsive mechanical stimulus. Acta Astronautica 43: 65-75, 1998. https://doi.org/10.1016/S0094-5765(98)00144-1

GOPALAKRISHNAN R, GENC KO, RICE AJ, LEE SM, EVANS HJ, MAENDER CC, ILASLAN H, CAVANAGH PR: Muscle volume, strength, endurance, and exercise loads during 6-month missions in space. Aviat Space Environ Med 81: 91-102, 2010. https://doi.org/10.3357/ASEM.2583.2010

GUILLIAMS ME, LEE SMC, SHEPHERD B, CHAUVIN J, TADDEO T, SHACKELFORD LC: Upper-body strength and endurance after long-duration spaceflight. Med Sci Sports Exercise 35 (Suppl 1): S262, 2003. https://doi.org/10.1097/00005768-200305001-01451

GUROVSKY NN, GAZENKO OG, ADAMOVICH BA, ILYIN EA, GENIN AM, KOROLKOV VI, SHIPOV AA, KOTOVSKAYA AR, KONDRATYEVA VA, SEROVA LV, KONDRATYEV YI: Study of physiological effects of weightlessness and artificial gravity in the flight of the biosatellite Cosmos-936. Acta Astronaut 7: 113-121, 1980. https://doi.org/10.1016/0094-5765(80)90122-8

HADDAD F, HERRICK RE, ADAMS GR, BALDWIN KM: Myosin heavy chain expression in rodent skeletal muscle: effects of exposure to zero gravity. J Appl Physiol (1985) 75: 2471-2477, 1993. https://doi.org/10.1152/jappl.1993.75.6.2471

HANSEN G, MARTINUK KJ, BELL GJ, MACLEAN IM, MARTIN TP, PUTMAN CT: Effects of spaceflight on myosin heavy-chain content, fibre morphology and succinate dehydrogenase activity in rat diaphragm. Pflugers Arch 448: 239-247, 2004. https://doi.org/10.1007/s00424-003-1230-9 
HENRIKSEN EJ, TISCHLER ME, WOODMAN CR, MUNOZ KA, STUMP CS, KIRBY CR: Elevated interstitial fluid volume in soleus muscles unweighted by spaceflight or suspension. J Appl Physiol (1985) 75: 1650-1653, 1993. https://doi.org/10.1152/jappl.1993.75.4.1650

HIDES JA, LAMBRECHT G, STANTON WR, DAMANN V: Changes in multifidus and abdominal muscle size in response to microgravity: possible implications for low back pain research. Eur Spine J 25 Suppl 1: 175-182, 2016. https://doi.org/10.1007/s00586-015-4311-5

HIGGINS J PT, GREEN S (Eds): Cochrane Handbook for Systematic Reviews of Interventions Version 5.1.0 [updated March 2011]. The Cochrane Collaboration.

HOLY X, MOUNIER Y: Effects of short spaceflights on mechanical characteristics of rat muscles. Muscle Nerve 14: 70-78, 1991. https://doi.org/10.1002/mus.880140112

JEE WS, WRONSKI TJ, MOREY ER, KIMMEL DB: Effects of spaceflight on trabecular bone in rats. Am J Physiol 244: R310-314, 1983. https://doi.org/10.1152/ajpregu.1983.244.3.R310

KEYAK JH, KOYAMA AK, LEBLANC A, LU Y, LANG TF: Reduction in proximal femoral strength due to longduration spaceflight. Bone 44: 449-453, 2009. https://doi.org/10.1016/j.bone.2008.11.014

KORYAK YA: Architectural and functional specifics of the human triceps surae muscle in vivo and its adaptation to microgravity. J Appl Physiol (1985) 126: 880-893, 2019. https://doi.org/10.1152/japplphysiol.00634.2018

KRAEMER WJ, STARON RS, GORDON SE, VOLEK JS, KOZIRIS LP, DUNCAN ND, NINDL BC, GÓMEZ AL, MARX JO, FRY AC, MURRAY JD: The effects of 10 days of spaceflight on the shuttle Endeavor on predominantly fast-twitch muscles in the rat. Histochem Cell Biol 114: 349-355, 2019. https://doi.org/10.1007/s004180000193

LALANI R, BHASIN S, BYHOWER F, TARNUZZER R, GRANT M, SHEN R, ASA S, EZZAT S, GONZALEZCADAVID NF: Myostatin and insulin-like growth factor-I and -II expression in the muscle of rats exposed to the microgravity environment of the NeuroLab space shuttle flight. J Endocrinol 167: 417-428, 2000. https://doi.org/10.1677/joe.0.1670417

LAMBERTZ D, PÉROT C, KASPRANSKI R, GOUBEL F: Effects of long-term spaceflight on mechanical properties of muscles in humans. J Appl Physiol (1985) 90: 179-188, 2001. https://doi.org/10.1152/jappl.2001.90.1.179

LANG T, LEBLANC A, EVANS H, LU Y, GENANT H, YU A: Cortical and trabecular bone mineral loss from the spine and hip in long-duration spaceflight. J Bone Miner Res 19: 1006-1012, 2004. https://doi.org/10.1359/JBMR.040307

LAUGHLIN MS, GUILLIAMS ME, NIESCHWITZ BA, HOELLEN D: Functional fitness testing results following long-duration ISS missions. Aerosp Med Hum Perform 86 (12 Suppl): A87-A91, 2015. https://doi.org/10.3357/AMHP.EC11.2015

LEBLANC A, SCHNEIDER V, SHACKELFORD L, WEST S, OGANOV V, BAKULIN A, VORONIN L: Bone mineral and lean tissue loss after long duration space flight. J Musculoskelet Neuronal Interact 1: 157-160, 2000 .

LI XT, YANG CB, ZHU YS, SUN J, SHI F, WANG YC, GAO Y, ZHAO JD, SUN XQ: Moderate exercise based on artificial gravity preserves orthostatic tolerance and exercise capacity during short-term head-down bed rest. Physiol Res 66: 567-580, 2017. https://doi.org/10.33549/physiolres.933493

LUCIANO TF, MARQUES SO, PIERI BL, DE SOUZA DR, ARAÚJO LV, NESI RT, SCHEFFER DL, COMIN VH, PINHO RA, MULLER AP, DE SOUZA CT: Responses of skeletal muscle hypertrophy in Wistar rats to different resistance exercise models. Physiol Res 66: 317-323, 2017. https://doi.org/10.33549/physiolres.933256

MACAULAY TR, SIAMWALA JH, HARGENS AR, MACIAS BR: Thirty days of spaceflight does not alter murine calvariae structure despite increased Sost expression. Bone Rep 7: 57-62, 2017. https://doi.org/10.1016/j.bonr.2017.08.004

MACK PB, LACHANCE PA, VOSE GP, VOGT FB: Bone demineralization of foot and hand of gemini-titan IV, V and VII astronauts during orbital flight. Am J Roentgenol Radium Ther Nucl Med 100(3): 503-511, 1967. https://doi.org/10.2214/ajr.100.3.503

MACK PB, VOGT FB: Roentgenographic bone density changes in astronauts during representative Apollo space flight. Am J Roentgenol Radium Ther Nucl Med 113: 621-633, 1971. https://doi.org/10.2214/ajr.113.4.621 
MARTIN TP, EDGERTON VR, GRINDELAND RE: Influence of spaceflight on rat skeletal muscle. J Appl Physiol (1985) 65: 2318-2325, 1988. https://doi.org/10.1152/jappl.1988.64.6.2318

MAUPIN KA, CHILDRESS P, BRINKER A, KHAN F, ABEYSEKERA I, AGUILAR IN, OLIVOS DJ 3RD, ADAM G, SAVAGLIO MK, GANESH V, GORDEN R, MANNFELD R, BECKNER E, HORAN DJ, ROBLING AG, CHAKRABORTY N, GAUTAM A, HAMMAMIEH R, KACENA MA: Skeletal adaptations in young male mice after 4 weeks aboard the International Space Station. NPJ Microgravity 5: 21, 2019. https://doi.org/10.1038/s41526-019-0081-4

MCNAMARA KP, GREENE KA, MOORE AM, LENCHIK L, WEAVER AA: Lumbopelvic muscle changes following long-duration spaceflight. Front Physiol 10: 627, 2019. https://doi.org/10.3389/fphys.2019.00627

MCNAMARA KP, GREENE KA, TOOZE JA, DANG J, KHATTAB K, LENCHIK L, WEAVER AA: Neck muscle changes following long-duration spaceflight. Front Physiol 10: 1115, 2019. https://doi.org/10.3389/fphys.2019.01115

MECHANIC GL, ARNAUD SB, BOYDE A, BROMAGE TG, BUCKENDAHL P, ELLIOTT JC, KATZ EP, DURNOVA GN: Regional distribution of mineral and matrix in the femurs of rats flown on Cosmos 1887 biosatellite. FASEB J 4: 34-40, 1990. https://doi.org/10.1096/fasebj.4.1.2295376

MIU B, MARTIN TP, ROY RR, OGANOV V, ILYINA-KAKUEVA E, MARINI JF, LEGER JJ, BODINE-FOWLER SC, EDGERTON VR: Metabolic and morphologic properties of single muscle fibers in the rat after spaceflight, Cosmos 1887. FASEB J 4: 64-72, 1990. https://doi.org/10.1096/fasebj.4.1.2136839

MOHER D, LIBERATI A, TETZLAFF J, ALTMAN DG; PRISMA GROUP: Preferred reporting items for systematic reviews and meta-analyses: the PRISMA statement. BMJ 339: b2535, 2009. https://doi.org/10.1136/bmj.b2535

MOREY ER, BAYLINK DJ: Inhibition of bone formation during space flight. Science 201: 1138-1141, 1978. https://doi.org/10.1126/science.150643

MUSACCHIA XJ, STEFFEN JM, FELL RD, DOMBROWSKI MJ: Skeletal muscle response to spaceflight, whole body suspension, and recovery in rats. J Appl Physiol (1985) 69: 2248-2253, 1990. https://doi.org/10.1152/jappl.1990.69.6.2248

OGANOV VS, RAKHMANOV AS, NOVIKOV VE, ZATSEPIN ST, RODIONOVA SS, CANN C: The state of human bone tissue during space flight. Acta Astronautica 23: 129-133, 1991. https://doi.org/10.1016/00945765(91)90109-I

OHIRA Y, JIANG B, ROY RR, OGANOV V, ILYINA-KAKUEVA E, MARINI JF, EDGERTON VR: Rat soleus muscle fiber responses to 14 days of spaceflight and hindlimb suspension. J Appl Physiol (1985) 73(2 Suppl): 51S-57S, 1992. https://doi.org/10.1152/jappl.1992.73.2.S51

PUGLIA I, BALSAMO M, VUKICH M, ZOLESI V: Long-term microgravity effects on isometric handgrip and precision pinch force with visual and proprioceptive feedback. Int J Aerospace Eng 2018: 1-11, 2018. https://doi.org/10.1155/2018/1952630

RADUGINA EA, ALMEIDA EAC, BLABER E, POPLINSKAYA VA, MARKITANTOVA YV, GRIGORYAN EN: Exposure to microgravity for 30 days onboard Bion M1 caused muscle atrophy and impaired regeneration in murine femoral Quadriceps. Life Sci Space Res (Amst) 16: 18-25, 2018. https://doi.org/10.1016/j.1ssr.2017.08.005

RAMBAUT PC, JOHNSTON RS: Prolonged weightlessness and calcium loss in man. Acta Astronautica 6: 1113-1122, 1979. https://doi.org/10.1016/0094-5765(79)90059-6

RICHARDSON WS, WILSON MC, NISHIKAWA J, HAYWARD RS: The well-built clinical question: a key to evidence-based decisions. ACP journal club 123: A12-13, 1995.

RILEY DA, BAIN JL, THOMPSON JL, FITTS RH, WIDRICK JJ, TRAPPE SW, TRAPPE TA, COSTILL DL: Decreased thin filament density and length in human atrophic soleus muscle fibers after spaceflight. J Appl Physiol (1985) 88: 567-572, 2000. https://doi.org/10.1152/jappl.2000.88.2.567

RILEY DA, ELLIS S, SLOCUM GR, SEDLAK FR, BAIN JL, KRIPPENDORF BB, LEHMAN CT, MACIAS MY, THOMPSON JL, VIJAYAN K, DE BRUIN JA: In-flight and postflight changes in skeletal muscles of SLS-1 and SLS-2 spaceflown rats. J Appl Physiol (1985) 81: 133-144, 1996. https://doi.org/10.1152/jappl.1996.81.1.133 
SANDONÀ D, DESAPHY JF, CAMERINO GM, BIANCHINI E, CICILIOT S, DANIELI-BETTO D, DOBROWOLNY G, FURLAN S, GERMINARIO E, GOTO K, GUTSMANN M, KAWANO F, NAKAI N, OHIRA T, OHNO Y, PICARD A, SALANOVA M, SCHIFFL G, BLOTTNER D, MUSARÒ A, OHIRA Y, BETTO R, CONTE D, SCHIAFFINO S: Adaptation of mouse skeletal muscle to long-term microgravity in the MDS mission. PLoS One 7(3): e33232, 2012. https://doi.org/10.1371/journal.pone.0033232

SCHUENKE MD, REED DW, KRAEMER WJ, STARON RS, VOLEK JS, HYMER WC, GORDON S, PERRY KOZIRIS L: Effects of 14 days of microgravity on fast hindlimb and diaphragm muscles of the rat. Eur J Appl Physiol 106: 885-892, 2009. https://doi.org/10.1007/s00421-009-1091-9

SHENKMAN BS, BELOZEROVA IN, LEE P, NEMIROVSKAYA TL, KOZLOVSKAYA IB: Effects of weightlessness and movement restriction on the structure and metabolism of the soleus muscle in monkeys after space flight. Neurosci Behav Physiol 33: 717-722, 2003. https://doi.org/10.1023/A:1024473126643

SMITH MCJ, RAMBAUT PC, VOGEL JM, WHITTLE MW: Biomedical Results from Skylab. 183-190, 1977.

SMITH SM, HEER M, SHACKELFORD LC, SIBONGA JD, SPATZ J, PIETRZYK RA, HUDSON EK, ZWART SR: Bone metabolism and renal stone risk during International Space Station missions. Bone 81: 712-720, 2015. https://doi.org/10.1016/j.bone.2015.10.002

SMITH SM, HEER MA, SHACKELFORD LC, SIBONGA JD, PLOUTZ-SNYDER L, ZWART SR: Benefits for bone from resistance exercise and nutrition in long-duration spaceflight: Evidence from biochemistry and densitometry. J Bone Miner Res 27(9): 1896-1906, 2012. https://doi.org/10.1002/jbmr.1647

SMITH SM, WASTNEY ME, O'BRIEN KO, MORUKOV BV, LARINA IM, ABRAMS SA, DAVIS-STREET JE, OGANOV V, SHACKELFORD LC: Bone markers, calcium metabolism, and calcium kinetics during extended-duration space flight on the mir space station. J Bone Miner Res 20: 208-218, 2005. https://doi.org/10.1359/JBMR.041105

SMITH SM, WASTNEY ME, MORUKOV BV, LARINA IM, NYQUIST LE, ABRAMS SA, TARAN EN, SHIH CY, NILLEN JL, DAVIS-STREET JE, RICE BL, LANE HW: Calcium metabolism before, during, and after a 3mo spaceflight: kinetic and biochemical changes. Am J Physiol 277: R1-10, 1999. https://doi.org/10.1152/ajpregu.1999.277.1.R1

TASCHER G, BRIOCHE T, MAES P, CHOPARD A, O'GORMAN D, GAUQUELIN-KOCH G, BLANC S, BERTILE F: Proteome-wide adaptations of mouse skeletal muscles during a full month in space. J Proteome Res 16: 2623-2638, 2017. https://doi.org/10.1021/acs.jproteome.7b00201

TESCH PA, BERG HE, BRING D, EVANS HJ, LEBLANC AD: Effects of 17-day spaceflight on knee extensor muscle function and size. Eur J Appl Physiol 93: 463-468, 2005. https://doi.org/10.1007/s00421-004-1236-9

THOMASON DB, MORRISON PR, OGANOV V, ILYINA-KAKUEVA E, BOOTH FW, BALDWIN KM: Altered actin and myosin expression in muscle during exposure to microgravity. J Appl Physiol (1985) 73 (2 Suppl): 90S-93S, 1992. https://doi.org/10.1152/jappl.1992.73.2.S90

THORNTON WE, RUMMEL JA: Biomedical Results from Skylab. NASA SP-377. NASA SP-377: 491, 1977.

TRAPPE S, COSTILL D, GALLAGHER P, CREER A, PETERS JR, EVANS H, RILEY DA, FITTS RH: Exercise in space: human skeletal muscle after 6 months aboard the International Space Station. J Appl Physiol (1985) 106: 1159-1168, 2009. https://doi.org/10.1152/japplphysiol.91578.2008

TRAPPE SW, TRAPPE TA, LEE GA, WIDRICK JJ, COSTILL DL, FITTS RH: Comparison of a space shuttle flight (STS-78) and bed rest on human muscle function. J Appl Physiol (1985) 91: 57-64, 2001. https://doi.org/10.1152/jappl.2001.91.1.57

VANDENBURGH H, CHROMIAK J, SHANSKY J, DEL TATTO M, LEMAIRE J: Space travel directly induces skeletal muscle atrophy. FASEB J 13: 1031-1038, 1999. https://doi.org/10.1096/fasebj.13.9.1031

VICO L, CHAPPARD D, PALLE S, BAKULIN AV, NOVIKOV VE, ALEXANDRE C: Trabecular bone remodeling after seven days of weightlessness exposure (BIOCOSMOS 1667). Am J Physiol 255(2 Pt 2): R243-247, 1988. https://doi.org/10.1152/ajpregu.1988.255.2.R243

VICO L, COLLET P, GUIGNANDON A, LAFAGE-PROUST MH, THOMAS T, REHAILLIA M, ALEXANDRE C: Effects of long-term microgravity exposure on cancellous and cortical weight-bearing bones of cosmonauts. The Lancet 355: 1607-1611, 2000. https://doi.org/10.1016/S0140-6736(00)02217-0 
VICO L, VAN RIETBERGEN B, VILAYPHIOU N, LINOSSIER MT, LOCRELLE H, NORMAND M, ZOUCH M, GERBAIX M, BONNET N, NOVIKOV V, THOMAS T, VASSILIEVA G: Cortical and trabecular bone microstructure did not recover at weight-bearing skeletal sites and progressively deteriorated at non-weightbearing sites during the year following international space station missions. J Bone Miner Res 32: 2010-2021, 2017. https://doi.org/10.1002/jbmr.3188

VOGEL JM: Bone mineral measurement: Skylab experiment M-078. Acta Astronautica 2: 129-139, 1975. https://doi.org/10.1016/0094-5765(75)90049-1

WHEDON GD, LUTWAK L: Metabolic studies of the Gemini-7 14 day orbital space flight. NASA (National Aeronautics and Space Administration), Scientific and Technical Aerospace Reports, 1971.

ZERATH E, NOVIKOV V, LEBLANC A, BAKULIN A, OGANOV V, GRYNPAS M: Effects of spaceflight on bone mineralization in the rhesus monkey. J Appl Physiol (1985) 81: 194-200, 1996. https://doi.org/10.1152/jappl.1996.81.1.194

ZERNICKE RF, VAILAS AC, GRINDELAND RE, KAPLANSKY A, SALEM GJ, MARTINEZ DA: Spaceflight effects on biomechanical and biochemical properties of rat vertebrae. Am J Physiol 258(6 Pt 2): R1327-1332, 1990. https://doi.org/10.1152/ajpregu.1990.258.6.R1327. 